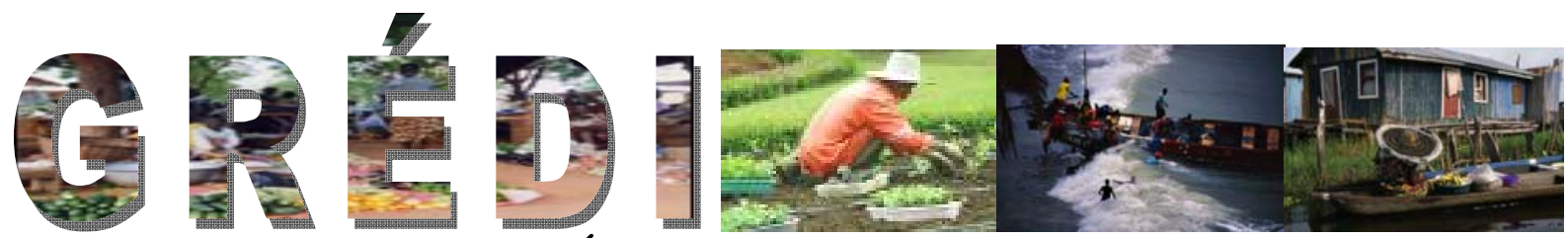

Groupe de Recherche en Économie et Développement International

Cahier de recherche / Working Paper

07-14

Une évaluation du rôle des déterminants du partage de la valeur ajoutée

Stéphane Mussard

Bernard Philippe 


\title{
Une évaluation du rôle des déterminants du partage de la valeur ajoutée
}

\author{
Stéphane Mussard* \\ CEPS/INSTEAD, GRÉDI, GEREM GEREM, Université de Perpignan
}

\begin{abstract}
Résumé
Au cours de la période 1961/2000, pourquoi la tendance à la hausse des taux de chômage s'est-elle inversée dans des économies «anglo-saxonnes » comme les États-Unis et le RoyaumeUni et pas dans des économies « continentales»telles que l'Allemagne, la France et l'Italie? Nous soutenons que cette inversion ne peut pas être imputée au fait que ces dernières auraient maîtrisé leurs coûts salariaux unitaires moins bien que les premières. Pour défendre ce point de vue, nous commençons par expliquer comment et pourquoi nous sommes incités à nous intéresser aux déterminants d'un indicateur statistique : le taux de croissance du taux de marge. Afin d'évaluer l'influence exercée sur ce taux par chacun de ses déterminants au cours de la période 1961-2000, nous utilisons la fonction valeur de Shapley de manière particulière : en tant qu'outil permettant de décomposer un indicateur diachronique déterminé par une structure multiplicative.
\end{abstract}

Mots-clés : Coût salarial unitaire, Décomposition, Shapley, Taux de chômage, Taux de marge.

Codes JEL : E25, E24, C39.

* Correspondance : CEPS/INSTEAD Luxembourg, GRÉDI Université de Sherbrooke, and GEREM, Département des Sciences Économiques - Adresse : GEREM, Université de Perpignan, 52 Avenue Paul Alduy, 66860 Perpignan Cedex, France, E-mail : smussard@adm.usherbrooke.ca. Ce papier a été en partie écrit lorsque j'étais post-doctorant au CEPS/INSTEAD Luxembourg. Je remercie le CEPS/INSTEAD, le Minsitère de la recherche du Luxembourg et Philippe Van Kerm.

${ }^{\dagger}$ GEREM, Département des Sciences Économiques, Université de Perpignan, E-mail : philippe@univ-perp.fr 


\section{Introduction}

En 1953, Shapley conçoit une fonction (la fonction valeur de Shapley) permettant d'expliquer comment un surplus, un bénéfice, une dette, un coût peut être «justement » réparti entre les agents économiques qui ont contribué à sa formation. En 1992, Auvray et Trannoy montrent que cette procédure d'évaluation peut être utilisée pour «mesurer» la contribution de chacun des facteurs considérés comme les déterminants d'une inégalité dont on connaît la mesure. Cette procédure est à nouveau employée dans le même champ par Chantreuil et Trannoy en 1999 puis par Sastre et Trannoy en 2001. En 1999, elle l'est aussi par Shorrocks mais, cette fois pour évaluer la contribution de facteurs considérés comme les déterminants d'une situation de pauvreté appréhendée à l'aide d'un indice. Silber (2004) confirme que la Valeur de Shapley est effectivement une très bonne technique de décomposition.

Dans les lignes qui suivent, nous privilégions cette procédure afin de mesurer, au cours de la période 1961/2000, la contribution des variables que nous considérons comme les déterminants des taux de croissance annuels des taux de marge de six économies : les États-Unis, l'Allemagne, le Royaume-Uni, la France, l'Italie et le Luxembourg.

Les résultats auxquels nous parvenons sont obtenus au terme d'une démarche qui peut être présentée en trois temps. Dans la première section de l'article nous expliquons pourquoi nous décidons de considérer le coût salarial moyen de l'unité d'excédent brut d'exploitation, le taux d'inflation et le taux de croissance du coût salarial de l'unité de valeur ajoutée comme les déterminants fondamentaux des taux de croissance des taux de marge. Bien que nous nous intéressions à un processus diachronique, bien que les valeurs prises par l'indicateur qui nous intéresse dépendent d'une structure multiplicative, nous montrons, dans la deuxième section, que la fonction valeur de Shapley peut être utilisée pour concevoir une décomposition linéaire fournissant une possibilité de mesure de la contribution de chacun de ces trois facteurs à la formation du taux de croissance annuel des taux de marge. ${ }^{1}$ Dans la troisième section nous appliquons cette technique de décomposition aux mesures des taux de croissance des taux de marge des six économies précitées. Dans la conclusion de l'article nous soulignons que nos résultats conduisent à partager les doutes déjà exprimés, par exemple, par des auteurs comme Blanchard (2001, 2004), Boussemart et alii (2000), ou Freyssinet et alii (2000) à propos de l'idée d'eurosclérose.

\footnotetext{
${ }^{1}$ La possibilité d'utiliser la valeur de Shapley pour décomposer un indicateur dont le montant dépend d'une structure multiplicative est aussi mise en évidence dans le travail de décomposition de l'indice de Malmquist que Mussard et Peypoch (2006) proposent afin de réfléchir à l'évolution de la productivité globale des facteurs.
} 


\section{Une mesure du taux de croissance du taux de marge}

L'évaluation à laquelle nous voulons procéder implique une identification : celle des variables auxquelles nous attribuons le statut de déterminant du partage de la valeur ajoutée. Nous en privilégions trois : le coût salarial de l'unité d'excédent brut d'exploitation, le taux d'inflation et le taux de croissance du coût salarial unitaire. Nous sommes parvenus à ce choix au terme d'une réflexion qui articule un constat, une suggestion et une opportunité.

Le constat est le suivant : dans l'état actuel de ses connaissances, la communauté des économistes ne parvient pas à démontrer que le partage de la valeur ajoutée dans les économies actuelles puisse être totalement indépendant des rapports de force entre agents économiques. Les économistes réussissent certes à concevoir deux types de représentations dans lesquelles ce type de rapport n’influence pas du tout le partage de la valeur ajoutée. Les premières n'impliquent pas la construction d'un agrégat nommé capital, il s'agit des modèles d'équilibre général walrasien. Les secondes impliquent au contraire la construction de cet agrégat et son utilisation comme argument d'une fonction de production de type $Y=F(K, L)$. L'emploi du premier type de représentation permet de conclure que le partage de la valeur ajoutée dépend de la signature d'accords exclusivement influencés par les fondamentaux de l'économie : préférences des agents, état des connaissances techniques, dotations initiales. L'emploi du second permet de soutenir que le montant du taux d'intérêt ou taux de profit et celui du taux de salaire sont égaux à des productivités marginales, elles mêmes exclusivement fonction de l'état des connaissances techniques et des préférences intertemporelles des agents. Ces conclusions concernent-elles le fonctionnement des économies actuelles? Non. Comme Clower et Howitt le soulignent les conclusions déduites du premier type de représentation concernent des économies virtuelles ${ }^{2}$. En effet, la réflexion que Arrow et Debreu consacrent à l'équilibre walrasien conduit à conclure que cet équilibre peut être conçu (existence) et atteint ${ }^{3}$ (convergence) pourvu que le futur ne soit pas radicalement incertain, pourvu que les directions d'entreprises ne supportent pas de coûts fixes et ne puissent bénéficier de rendements d'échelle croissants, pourvu qu'un commissaire priseur puisse identifier les composantes du vecteur prix garantissant la compatibilité des plans avant que les agents se livrent au moindre échange ou à la moindre production, etc. A l'évidence les économies dans lesquelles ces conditions sont respectées sont inassimilables aux économies actuelles. Comme Samuelson ${ }^{4}$ l'a reconnue, l'assimilation de la productivité marginale du capital

\footnotetext{
${ }^{2}$ Clower et Howitt (1995), p. 31-35.

${ }^{3}$ Ce qui n'est pas sûr. En effet la convergence vers l'équilibre walrasien est garantie si les plans dont le commissaire priseur recherche la compatibilité vérifient la propriété de substituabilité brute. Or, confer le théorème de Sonnenschein-Mantel-Debreu, la rationalité prêtée aux agents n'implique pas le respect de cette condition. Sur ce point confer Guerrien (1989).

${ }^{4}$ « Je suis reconnaissant au professeur Piero Garegnani ... de m'avoir évité d'affirmer une proposition fausse, à
} 
au taux d'intérêt à laquelle conduit le deuxième type de représentation n'est concevable que dans des économies qui méritent, elles aussi, d'être qualifiées de virtuelles. En effet ces économies sont celles dont les frontières des prix des facteurs peuvent être représentées sous forme linéaire car tous leurs secteurs productifs sont caractérisés par le même rapport entre capital et travail, c'est-à-dire par le même degré de mécanisation ${ }^{5}$.

La suggestion est inspirée par une analogie entre les sociétés féodales et les sociétés capitalistes. Dans les premières, la noblesse et le clergé étaient des ordres qui avaient droit à une fraction de la valeur ajoutée directement créée par un seul type de travail : celui des «laborantes ». Ces derniers ne contestèrent pas ce droit de manière systématique. N'était-il pas la contrepartie des obligations de protection physique et morale pesant sur les deux autres ordres? La détermination du montant du prélèvement auquel il donnait naissance, soit sous forme de corvées soit sous forme d'impôts, fut par contre souvent le résultat de conflits, de rapports de force dont l'Histoire porte témoignage. Dans les économies capitalistes trois rôles privés peuvent être distingués : celui d'apporteur de capitaux, celui de dirigeant, celui d'exécutant. Ces rôles sont concaténés par une relation hiérarchique ${ }^{6}$ : les exécutants mettent en oeuvre les stratégies conçues par les dirigeants au nom des intérêts des apporteurs de capitaux. Dans le cadre de cette relation, l'apport de capital n'implique aucune participation directe à l'activité productive. Ceci n'exclut pas que cet apport, jugé fondamental, soit rémunéré. Les économistes ont-ils intérêt à admettre que le montant de ce type de rémunération dépend seulement d'interactions entre les fondamentaux? Non, puisque, nous venons le rappeler, les conditions à respecter pour pouvoir adopter ce point de vue sont invraisemblables. Dès lors pourquoi ne pas supposer que, dans les économies actuelles, la détermination du montant de la rémunération des apporteurs de capitaux dépend, comme celle de celui que percevaient la noblesse et le clergé, d'un prélèvement sur la valeur ajoutée? La référence à l'Histoire économique, du 19ième par exemple, accrédite l'intérêt de l'hypothèse. Le recours à l'idée bien connue selon laquelle les prix peuvent être fixés par des directions d'entreprise appliquant à leurs coûts moyens des taux de marge

savoir que mon hypothèse extrême concernant la proportionnalité des coefficients techniques dans les secteurs de consommation et d'investissement pouvait être retirée sans affecter la plupart des propositions subrogées. J'espère qu'il publiera ses commentaires où il montre pourquoi le cas subrogé est si particulier », cité par Lavoie (1987), p. 20. Toujours à propos du problème que pose la construction de l'agrégat capital, Hahn, l'un des néo-classiques les plus prestigieux n'hésita pas à conclure en 1975 : «Les néo-ricardiens, par l'intermédiaire du choix des techniques, ont établi que l'agrégation du capital était spécieuse. Parfait donnons leur un A pour ceci. », cité par Lavoie (1987), p. 99 De manière plus générale, à l'issue des controverses entre les Cambridge, il fut reconnu que l'existence du capital fixe excluait aussi bien l'assimilation de la productivité marginale à un taux que la construction de la fonction caractéristique d'une économie et la mesure de la durée de sa période de production.

${ }^{5}$ Sur ce point, confer la première partie de Lavoie (1987).

${ }^{6}$ Cf. Fleurbaey (2006, p. 123-136) pour une critique concernant les inégalités de pouvoir de décision au sein des entreprises et le recours au principe démocratique afin de les diminuer. 
fonction de leur pouvoir de monopole ${ }^{7}$ permet sa mise en oeuvre ${ }^{8}$.

L'opportunité nous est offerte par les résultats du travail des comptables nationaux. Pour chaque année, ces comptables évaluent, en respectant des conventions clairement définies, des grandeurs telles que la valeur ajoutée créée et l'excédent brut d'exploitation. Par définition, cet excédent représente la part de la valeur ajoutée qui n'est pas consacrée à la rémunération des agents qui, soit en tant qu'exécutants soit en tant que dirigeants, ont assumé des rôles de producteurs. A ce titre, il peut être assimilé au prélèvement effectué par les apporteurs de capitaux. Les comptables nationaux nomment taux de marge le rapport entre le montant de cet excédent et celui de la valeur ajoutée. Il est évident que le prélèvement des apporteurs de capitaux et ce taux varient dans le même sens. Si nous parvenions à identifier les facteurs qui déterminent le taux de croissance de ce taux nous disposerions bien d'une identification des déterminants des partages des valeurs ajoutées. Nous obtenons ce résultat de la manière suivante.

Pour les comptables nationaux l'excédent annuel nommé excédent brut d'exploitation $(E B E)$ est égal à la différence entre deux montants : celui de la valeur ajoutée globale créée au cours de la période $T$, telle qu'elle peut-être mesurée à la fin de cette période ou instant $t\left(V A_{t}\right)$, et celui de la somme des salaires versés au cours de la même période et lui aussi mesuré à l'instant $t\left(S_{t}\right)$ :

$$
E B E_{t}=V A_{t}-S_{t}
$$

A l'instant $t$, le taux de marge ou excédent brut d'exploitation par unité de valeur ajoutée créé en $T$ est donc mesuré par :

$$
T X M_{t}=E B E_{t} / V A_{t}=1-S_{t} / V A_{t} .
$$

Soit à l'instant $t: p_{t}$ la mesure du déflateur du PIB au cours de la période $T ; N_{t}$ la mesure de l'effectif employé au cours de $T$ pour produire ce $P I B ; P M R T_{t}$ la mesure de la productivité

\footnotetext{
${ }^{7}$ Supposons que les coûts supportés par une direction dépendent seulement de la valeur des consommations intermédiaires représentée par $C i$, de celle des salaires versés représentée par $S$, de celle des provisions constituées afin d'amortir l'usure du capital fixe représentée par $A m$. Supposons que par le biais de la vente de l'output $Q$, la direction revendique une recette $p Q$ telle que $p Q=C i+S+A m$. La valeur ajoutée nette qu'elle obtient si elle vend $Q$ est mesurée par $V A=p Q-C i-A m$. $V A$ est égale à $S$ et correspond à la rémunération des heures de travail fournies. En fait, en économie capitaliste $p$ est fixé en appliquant un taux de marge à $C i+S+A m$ de manière à ce que $p Q=C i+S+A m+B^{*}$, avec $B^{*}$ bénéfice ou profit espéré. En cas de vente de l'intégralité de la production la valeur ajoutée est donc, toujours mesurée par $V A=p Q-C i-A m$, mais elle est égale à $S+B^{*}$. En économie capitaliste le mode de fixation des prix joue donc bien un rôle similaire à celui de la corvée et des impôts dans les sociétés féodales : il autorise le prélèvement d'une partie de la valeur ajoutée en faveur des apporteurs de capitaux.

${ }^{8}$ Privilégier ce type de perspective n'est pas novateur. Dans son «Essai sur l'économie de Marx », publié pour la première fois en 1941, Joan Robinson (1971, p.14) s'intéresse au concept d'exploitation. Convaincue que « la transformation s'effectue des prix vers les valeurs et non en sens inverse », elle note : « La possibilité d'exploitation dépend de l'existence d'une marge entre le produit net et le minimum de subsistance des travailleurs. Si un travailleur ne peut produire plus dans un jour qu'il n'est obligé de manger, il n'est pas un objet potentiel d'exploitation. L'idée est simple et peut être exprimée en langage courant sans l'appareil d'une terminologie spéciale. Mais ce sont précisément ces caractéristiques simples et fondamentales du capitalisme qui ont été perdues de vue dans les sentiers tortueux de l'analyse économique universitaire. »
} 
moyenne réelle de chacune des unités de cet effectif au cours de $T$; $s_{t}$ la mesure du taux de salaire nominal moyen versé au cours de $T$ à chacun de ces unités. Puisque

$$
\begin{aligned}
V A_{t} & =p_{t} \cdot P M R T_{t} \cdot N_{t}, \\
E B E_{t} & =p_{t} \cdot P M R T_{t} \cdot N_{t}-s_{t} \cdot N_{t},
\end{aligned}
$$

le taux de marge mesuré à l'instant $t$ peut être réécrit :

$$
T X M_{t}=1-\frac{s_{t}}{p_{t} \cdot P M R T_{t}} .
$$

Afin de trouver une forme analytique du taux de croissance du taux de marge, commençons par supposer que le temps est continu. Nous obtenons :

$$
d T X M_{t}=-\frac{1}{p_{t-1} \cdot P M R T_{t-1}} d s_{t}+\frac{s_{t-1} \cdot P M R T_{t-1}}{\left(p_{t-1} \cdot P M R T_{t-1}\right)^{2}} d p_{t}+\frac{s_{t-1} \cdot p_{t-1}}{\left(p_{t-1} \cdot P M R T_{t-1}\right)^{2}} d P M R T_{t}
$$

avec $d T X M_{t}, d s_{t}, d p_{t}$ et $d P M R T_{t}$ variations de $T X M$, de $s$, de $p$ et de $P M R T$ entre les instants $t-1$ et $t$. Soit $\Delta$ l'opérateur donnant la différence entre une variable et elle-même retardée d'un pas de temps. Dans le cas discret, la différentielle totale peut alors être considérée comme une mesure approchée de la variation du taux de marge entre le début et la fin de $T$. Autrement dit, nous posons :

$$
\Delta T X M_{t}=T X M_{t}-T X M_{t-1} \cong d T X M_{t} .
$$

Par conséquent, après quelques manipulations algébriques, le taux de croissance du taux de marge s'écrit :

$$
\frac{\Delta T X M_{t}}{T X M_{t-1}}=\frac{s_{t-1}}{p_{t-1} \cdot P M R T_{t-1}-s_{t-1}}\left(\frac{\Delta p_{t}}{p_{t-1}}-\left(\frac{\Delta s_{t}}{s_{t-1}}-\frac{\Delta P M R T_{t}}{P R M T_{t-1}}\right)\right) .
$$

On simplifie l'expression en posant :

$$
\begin{aligned}
A_{t-1} & :=\frac{s_{t-1}}{p_{t-1} \cdot P M R T_{t-1}-s_{t-1}} \\
B_{t} & :=\frac{\Delta p_{t}}{p_{t-1}} \\
C_{t} & :=\frac{\Delta s_{t}}{s_{t-1}}-\frac{\Delta P M R T_{t}}{P R M T_{t-1}}
\end{aligned}
$$

On obtient ainsi une décomposition multiplicative du taux de croissance du taux de marge en trois composantes :

$$
\operatorname{TCTX} M_{t}:=\frac{\Delta T X M_{t}}{T X M_{t-1}}=A_{t-1}\left(B_{t}-C_{t}\right) .
$$

Dans la suite de notre exposé, nous décidons de considérer cette décomposition comme la mesure du taux de croissance annuel du taux de marge $(T C T X M)^{9}$. L'adoption de cette convention permet

\footnotetext{
${ }^{9}$ Dans l'Annexe, note 22 page 19, nous soulignons qu'il est aisé de vérifier que cette mesure mérite d'être considérée comme une mesure approchée satisfaisante de la « vraie» mesure.
} 
d'admettre, comme nous le supposions dans l'introduction, qu'entre l'instant $t-1$, qui marque la fin de l'année $T-1$ et le début de l'année $T$, et l'instant $t$, qui marque la fin de l'année $T$, le taux de croissance du taux de marge d'une économie est une grandeur déterminée par trois facteurs ${ }^{10}$. Le premier $A_{t-1}$ est le coût salarial moyen de l'excédent brut d'exploitation dégagé par unité de travail au cours de $T-1$; le second $B_{t}$ est le taux d'inflation mesuré entre la fin de l'année $T-1$ et celle de l'année $T$; le troisième $C_{t}$ est le taux de croissance du coût salarial unitaire lui aussi mesuré entre la fin de l'année $T-1$ et celle de l'année $T$ :

$$
C_{t}=\frac{\Delta C S U_{t}}{C S U_{t-1}}=\frac{\Delta s_{t}}{s_{t-1}}-\frac{\Delta P M R T_{t}}{P R M T_{t-1}}
$$

Il est possible de supposer que le terme $B_{t}$ est lié au taux de croissance du coût salarial unitaire par l'intermédiaire du comportement de «Mark-up ». La variable $B_{t}$ peut donc être considérée comme une variable d'ajustement à la disposition des chefs d'entreprise. En fixant cette variable, ces dirigeants peuvent essayer de contrecarrer les effets négatifs que les variations de $C$ peuvent exercer sur $\Delta T X M_{t} / T X M_{t-1}$. En d'autres termes, lorsque le taux de croissance du coût salarial augmente, les prix augmentent ${ }^{11}$. Le taux de croissance du taux de marge est donc représenté par une relation multiplicative où $A_{t-1}, B_{t}$ et $C_{t}$ interagissent. La perspective ouverte par Shapley (1953) permet de proposer une décomposition linéaire de cette mesure.

\section{La décomposition du taux de croissance du taux de marge par la valeur de Shapley}

\subsection{La valeur de Shapley}

La valeur de Shapley ( $C f$. Shapley (1953)) est un concept provenant de la géométrie des simplexes. Appliquée en théorie des jeux coopératifs, lorsque $p$ agents ou groupes sont identifiés, la valeur de Shapley permet d'expliciter les modalités de partage d'une somme (bénéfice, coût, etc.) notée $I$. Afin d'allouer à chaque agent tout ou partie de cette somme, on considère que les agents ont la possibilité de se coaliser. Soit $K$ la grande coalition, autrement dit, l'ensemble constitué des $p$ joueurs. Soit $k$ l'un de ces joueurs. Supposons que $k$ abandonne la coalition $K$. Soit $v$ le cardinal de l'ensemble obtenu après cet abandon : c'est-à-dire le cardinal de $\mathcal{V}$ tel que $\mathcal{V}:=K \backslash\{k\}$. En

\footnotetext{
${ }^{10}$ Soulignons que le montant de chacun des trois facteurs est lui-même déterminé par d'autres facteurs. Le montant de $A_{t-1}$ dépend des valeurs prises au cours de $T-1$ de $p, s$ et $P M R T$. A la fin de la période $T-1$ c'est-à-dire au début de la période $T$, ces valeurs sont héritées du passé, elles constituent un legs s'imposant aux directions des entreprises. Au début de la période $T$, ces directions peuvent par contre s'efforcer de maîtriser les déterminants du montant de $B_{t}$ et celui du montant de $C_{t}$, c'est-à-dire le montant de $p$, celui de $s$ et celui de $P M R T$ durant la période $T$.

${ }^{11}$ La qualité des régressions qui peuvent être identifiées pour les cinq économies entre le taux d'inflation en tant que variable expliquée et le taux de croissance du coût salarial unitaire en tant que variable explicative semble confirmer l'intérêt de ce point de vue.
} 
mesurant la différence entre la somme à se partager $I$ lorsque tous les joueurs se coalisent et la somme obtenue après le retrait du joueur $k$, on définit l'un des impacts marginaux associé au joueur $k$. On peut ensuite mesurer un autre impact en considérant que plusieurs joueurs se retirent à la fois. Soit l'ensemble de tous les ensembles mouvants $\mathcal{V}$ obtenus en tenant compte soit de la totalité, soit d'une partie ou bien d'aucun des éléments de $K$. La relation $\mathcal{V} \subseteq K$ étant toujours vérifiée, il est possible de représenter le processus d'élimination des joueurs pour le partage de $I$ à l'aide des formes fonctionnelles $F(\mathcal{V})$ définies par $F:\{\mathcal{V}: \mathcal{V} \subseteq K\} \longrightarrow \mathbb{R}$. Ces formes permettent de mesurer la contribution ou la part de $k$ au partage de $I$. Cette contribution, soit $\mathcal{C}^{k}$, est donnée par :

$$
\mathcal{C}^{k}(K ; F)=\sum_{v=0}^{p-1} \sum_{\mathcal{V} \subseteq K \backslash\{k\}} \frac{(p-1-v) ! v !}{p !} \Delta_{k} F(\mathcal{V}),
$$

où

$$
\Delta_{k} F(\mathcal{V}):=F(\mathcal{V} \cup\{k\})-F(\mathcal{V})
$$

En additionnant toutes les contributions $\mathcal{C}^{k}$, on retrouve la somme à se partager :

$$
F(K):=I=\sum_{k=1}^{p} \mathcal{C}^{k}
$$

\subsection{La valeur Nested Shapley}

Afin de « décomposer » les mesures d'inégalité, Auvray et Trannoy (1992) proposent une extension de la notion de valeur de Shapley nommée valeur « Nested » Shapley. Leur démarche est reprise par Chantreuil et Trannoy (1999), par Sastre et Trannoy (2002), par Silber (2004). Elle l'est aussi par Shorrocks (1999) qui l'applique aux mesures de pauvreté. Dans le cadre de cette démarche, l'ensemble $K$ ne représente plus l'ensemble des joueurs susceptibles de participer à une coalition mais l'ensemble des variables sensées déterminer le montant d'un indicateur statistique I. Le problème abordé est celui de la mesure de la contribution de chacun des $p$ éléments de $K$ à la détermination du montant de $I$. Il est résolu en substituant à l'idée d'abandon d'une coalition par un joueur celle d'élimination d'une variable par attribution à cette variable de la valeur zéro. Cette substitution s'avère compatible avec des procédures d'identification des ensembles mouvants $\mathcal{V}$, d'identification des formes fonctionnelles et de mesure des contributions identiques à celles qui sont employées lorsqu'on utilise la valeur de Shapley.

\subsection{La décomposition du taux de croissance du taux de marge}

Décomposer le taux de croissance du taux de marge à l'aide de la valeur de Shapley c'est procéder à la décomposition d'une mesure dont le montant dépend des résultats de stratégies adoptées au 
cours de deux périodes successives. La méthodologie que nous allons employer pour réaliser cette opération est de même type que celles que nous venons d'évoquer dans les deux points précédents.

Illustrons notre propos. La mesure, grâce à la valeur de Shapley, de la contribution de $A_{t-1}$, de celle de $B_{t}$ et de celle de $C_{t}$ à la détermination du taux de croissance du taux de marge en $t\left(T C T X M_{t}\right)$ implique d'une part le calcul de différences (impacts marginaux), d'autre part le calcul de moyennes pondérées (contributions). Les différences à calculer peuvent elles-mêmes être identifiées en remarquant que les stratégies mises en oeuvre par les agents peuvent engendrer ou ne pas engendrer des variations des variables considérées comme les déterminants de TCTXM.

\subsubsection{Une hypothèse sur les éliminations et les impacts marginaux}

Jusqu'ici nous nous sommes contentés de remarquer que la valeur prise chaque année par TCTXM dépendait de l'influence exercée par la valeur de $A_{t-1}$, celle de $B_{t}$ et par celle de $C_{t}$. Imaginons maintenant que nous ayons le droit de supposer que chacune de ces valeurs puisse être ou ne pas être nulle. Soit $\mathbb{H}$ cette hypothèse. Nous montrerons ci-dessous qu'elle est acceptable.

Si $A_{t-1}$ est nul, la détermination de $T C T X M$, au lieu d'être représentée par une fonction de type $T C T X M=F\left(A_{t-1}, B_{t}, C_{t}\right)$, peut l'être par une fonction de type $\operatorname{TCTXM}=F\left(B_{t}, C_{t}\right)$. Cette substitution autorise la mesure de la différence $F\left(A_{t-1}, B_{t}, C_{t}\right)-F\left(B_{t}, C_{t}\right)$. Cette différence peut être considérée comme une mesure de l'influence exercée par $A_{t-1}$ dans le processus de détermination de TCTXM. Suffit-elle à mesurer toute l'influence exercée par $A_{t-1}$ dans ce processus ? La réponse est non. Un exemple suffit à le prouver. Supposons que $C_{t}$ soit nul. La détermination de TCTXM est alors représentée par une fonction de type $\operatorname{TCTXM}=F\left(A_{t-1}, B_{t}\right)$. Supposons que $A_{t-1}$ et $C_{t}$ soient simultanément nuls. La détermination de TCTXM est alors représentée par une fonction de type TCTXM=F(Bt). La mesure de la différence $F\left(A_{t-1}, B_{t}\right)-F\left(B_{t}\right)$ doit, elle aussi, être considérée comme une mesure de l'influence exercée par $A_{t-1}$, autrement dit, comme la mesure d'un impact marginal associé à $A_{t-1}$. A travers combien d'impacts marginaux l'influence de $A_{t-1}$ s'exercet-elle? Qu'en est-il pour $B_{t}$ et $C_{t}$ ? Quelle importance accorder à chaque impact dans la mesure de la contribution globale exercée par $A_{t-1}, B_{t}, C_{t}$ ? Il est possible de répondre à ces questions en s'intéressant à l'arbre suivant. 
Figure 1 : Le processus de décomposition du taux de croissance du taux de marge

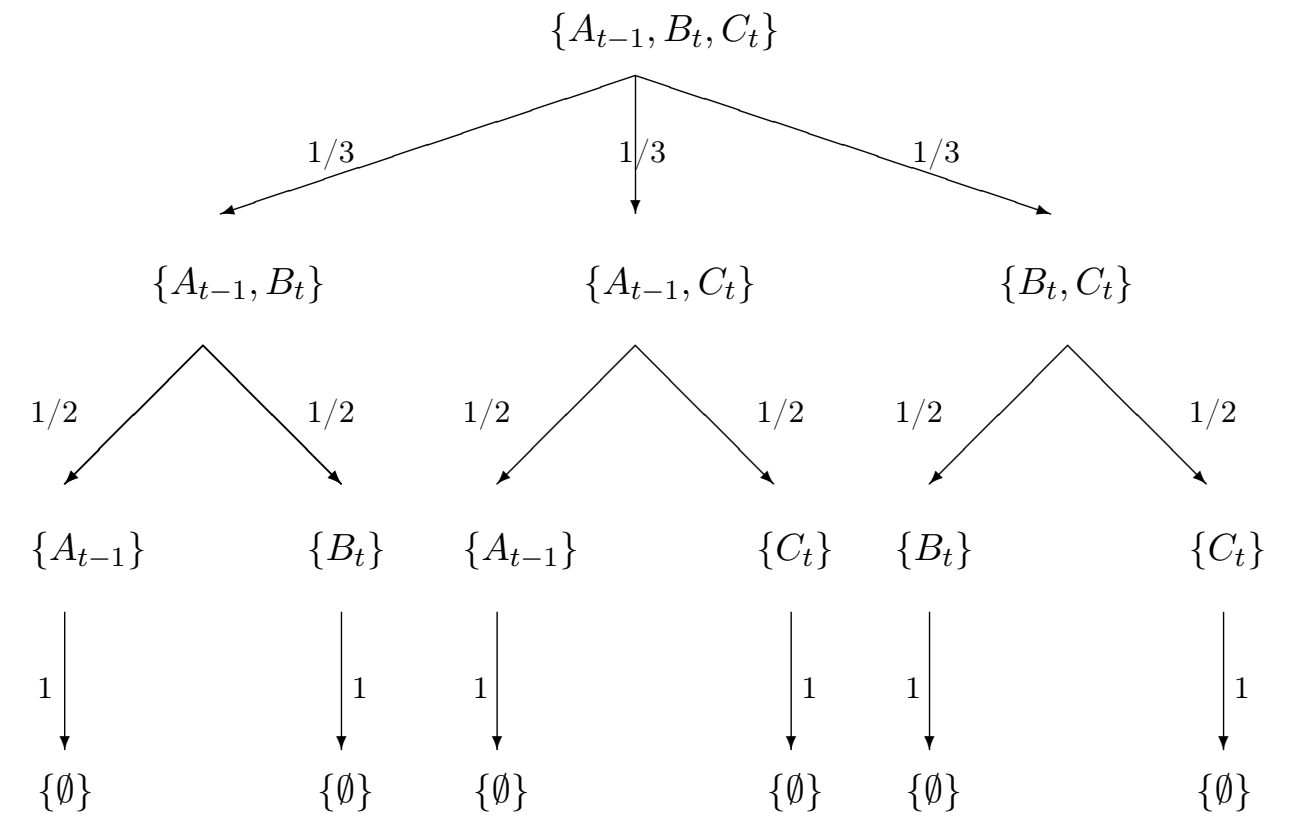

Dans cet arbre, les probabilités sont identifiées au terme d'un raisonnement de ce type : si je décide d'éliminer un des trois facteurs de l'ensemble $\{A, B, C\}$, j'ai une chance sur trois d'obtenir l'ensemble $\{A, B\}$, une sur trois d'obtenir l'ensemble $\{A, C\}$, etc. Si je décide d'éliminer un des deux facteurs de l'ensemble $\{A, B\}$, j'ai une chance sur deux d'obtenir l'ensemble $\{A\}$, une chance sur deux d'obtenir l'ensemble $\{B\}$, etc. Si je décide d'éliminer un facteur de l'ensemble $\{A\}$, j'ai cent pour cent de chance d'obtenir l'ensemble vide, etc.

\subsubsection{H : une hypothèse acceptable}

La mesure de TCTXM que nous avons adoptée nous permet de soutenir que son montant dépend chaque année de la variation de $s$, de celle de $p$ et de celle de $P M R T$ d'une part entre $t-2$ et $t-1$, d'autre part entre $t-1$ et $t$. Ces variations peuvent elles-mêmes être considérées comme des grandeurs qui traduisent l'efficacité des stratégies concernant soit les relations humaines au sein des entreprises, soit la qualité de l'insertion de ces entreprises dans leur environnement. Les premières contribuent à la détermination des taux de salaire et, via la tolérance des salariés à l'égard de leurs conditions de travail, à la détermination de $P M R T$. Les secondes, dans la mesure où elles déterminent, entre autres, les montants des ventes et l'importance du pouvoir de monopole dont dispose chaque entreprise, contribuent à la fixation de $P M R T$ et de $p$. Il est possible d'envisager que l'efficacité de ces stratégies entre $t-2$ et $t-1$ soit telle que $s_{t-2}=s_{t-1}, p_{t-2}=p_{t-1}, P M R T_{t-2}=$ $P M R T_{t-1}$. La même démarche permet d'envisager que $s_{t-1}=s_{t}$, que $p_{t-1}=p_{t}$ et que $P M R T_{t-1}=$ 
$P M R T_{t}$. Autrement dit, nous pouvons imaginer que les variations de $s$, de $p$ et de $P M R T$ soient nulles aussi bien entre $t-2$ et $t-1$, qu'entre $t-1$ et $t$. Dans ces conditions :

si $s_{t-2}=s_{t-1}, p_{t-2}=p_{t-1}, P M R T_{t-2}=P M R T_{t-1}$ alors $A_{t-1}=A_{t-2}$;

si $p_{t-1}=p_{t}$ alors $B_{t}=0$;

si $s_{t-1}=s_{t}$ et $P M R T_{t-1}=P M R T$ alors $C_{t}=0$.

En définitive, pour des mesures diachroniques, il est possible de substituer à l'hypothèse de nullité des variables imposée dans la procédure Nested Shapley, celle de nullité des variations des variables considérées. Ainsi, nous pouvons expliciter les formes fonctionnelles permettant la mesure des impacts marginaux, donc celle du taux de croissance du taux de marge.

Proposition 3.1 La décomposition par la valeur de Shapley du taux de croissance du taux de marge, défini par le schéma multiplicatif unissant les variables $A_{t-1}, B_{t}, C_{t}$, procure une décomposition linéaire permettant de mesurer la contribution de chaque variable au montant global du taux de croissance du taux de marge tel qu'il peut être mesuré à la fin de chaque année.

Preuve : En l'absence de variations des variables dont les mesures $A_{t-1}, B_{t}$ et $C_{t}$ dépendent, nous obtenons les conditions suivantes :

$$
\begin{aligned}
& \Delta s_{t-1}=0, \Delta P M R T_{t-1}=0, \Delta p_{t-1}=0 \Longrightarrow A_{t-1}=A_{t-2} ; \\
& \Delta p_{t}=0 \Longrightarrow B_{t}=0 ; \\
& \Delta P M R T_{t}=0, \Delta s_{t}=0 \Longrightarrow C_{t}=0 .
\end{aligned}
$$

Par conséquent, la séquence des formes fonctionnelles définissant le taux de croissance du taux de marge est la suivante :

$F(A, B, C)=A_{t-1}\left(B_{t}-C_{t}\right)$ si $A_{t-1} \neq A_{t-2}$, si $B_{t} \neq 0$, et si $C_{t} \neq 0 ; F(B, C)=A_{t-2}\left(B_{t}-\right.$ $\left.C_{t}\right)$ si $A_{t-1}=A_{t-2} ; F(A, C)=A_{t-1}\left(-C_{t}\right)$ si $B_{t}=0 ; F(A, B)=A_{t-1}\left(B_{t}\right)$ si $C_{t}=0 ; F(C)=$ $A_{t-2}\left(-C_{t}\right)$ si $A_{t-1}=A_{t-2}$ et si $B_{t}=0 ; F(B)=A_{t-2}\left(B_{t}\right)$ si $A_{t-1}=A_{t-2}$ et si $C_{t}=0 ; F(A)=$ 0 si $B_{t}=C_{t}=0 ; F(\emptyset)=0$ si $A_{t-1}=A_{t-2}$ et si $B_{t}=C_{t}=0$. Puisque toutes les formes fonctionnelles sont déterminées, les contributions des variables $A_{t-1}, B_{t}$ et $C_{t}$ peuvent être calculées :

$$
\begin{aligned}
\mathcal{C}^{A}= & 2 \frac{1}{6}\left[F\left(A_{t-1}\right)-F(\emptyset)\right]+\frac{1}{6}\left[F\left(A_{t-1}, B_{t}\right)-F\left(B_{t}\right)\right] \\
& +\frac{1}{6}\left[F\left(A_{t-1}, C_{t}\right)-F\left(C_{t}\right)\right]+1 / 3\left[F\left(A_{t-1}, B_{t}, C_{t}\right)-F\left(B_{t}, C_{t}\right)\right], \\
\mathcal{C}^{B}= & 2 \frac{1}{6}\left[F\left(B_{t}\right)-F(\emptyset)\right]+\frac{1}{6}\left[F\left(A_{t-1}, B_{t}\right)-F\left(A_{t-1}\right)\right] \\
& +\frac{1}{6}\left[F\left(B_{t}, C_{t}\right)-F\left(C_{t}\right)\right]+1 / 3\left[F\left(A_{t-1}, B_{t}, C_{t}\right)-F\left(A_{t-1}, C_{t}\right)\right], \\
\mathcal{C}^{C}= & 2 \frac{1}{6}\left[F\left(C_{t}\right)-F(\emptyset)\right]+\frac{1}{6}\left[F\left(A_{t-1}, C_{t}\right)-F\left(A_{t-1}\right)\right] \\
& +\frac{1}{6}\left[F\left(B_{t}, C_{t}\right)-F\left(B_{t}\right)\right]+1 / 3\left[F\left(A_{t-1}, B_{t}, C_{t}\right)-F\left(A_{t-1}, B_{t}\right)\right] .
\end{aligned}
$$


La preuve semble établie. Toutefois, rien ne nous garantit le fait que la somme des contributions des composantes $A_{t-1}, B_{t}$ et $C_{t}$ soit égale au taux de croissance du taux de marge. Or, étant donné que les différentes formes fonctionnelles $F(\emptyset)$ sont bien égales à zéro dans le cas que nous étudions, on peut vérifier que la somme des contributions mesurées par (17), (18), et (19) donne exactement la mesure du taux de croissance du taux de marge. Ce résultat est fondamental puisque, comme le soulignent Chantreuil et Trannoy (1999), la décomposition par la valeur de Shapley n'est une règle cohérente que si la somme des contributions de chaque variable procure exactement la statistique globale de départ : $F(K)=\sum_{k=1}^{p} \mathcal{C}^{k}$. Ceci nous permet de mesurer la contribution en pourcentage des variables $A_{t-1}, B_{t}$ et $C_{t}$ en vérifiant que leur somme est bien égale à $100 \%$. Si au contraire, notre résultat avait été respectivement strictement sur-cohérent ou strictement sous-cohérent, c'est-à-dire si nous avions eu :

$$
F(K)>\sum_{k=1}^{p} \mathcal{C}^{k} ; F(K)<\sum_{k=1}^{p} \mathcal{C}^{k}
$$

nous n'aurions pas pu décomposer le taux de croissance du taux de marge.

\section{Application}

Appliquée aux États-Unis, à l'Allemagne, au Royaume-Uni, à la France, à l'Italie et au Luxembourg, à propos de la période 1961/2000, la décomposition du taux de croissance annuel du taux de marge par la fonction valeur de Shapley conduit à la quantification des contributions des facteurs $A_{t-1}, B_{t}$ et $C_{t}$ proposée en Annexe. Les informations essentielles auxquelles cette quantification donne accès peuvent être présentées à l'aide du tableau 1.

Tableau 1 : Contributions des éléments $A, B$ et $C$

\begin{tabular}{lcccc}
\hline $1963 / 2000$ & $\mathcal{C}^{A}$ & $\mathcal{C}^{B}$ & $\mathcal{C}^{C}$ & TCTXM \\
\hline États-Unis & 0,00579 & 9,498 & $-9,294$ & 0,2098 \\
Allemagne & $-0,026$ & 8,801 & $-8,4905$ & 0,2845 \\
Royaume-Uni & 0,0884 & 13,527 & $-13,438$ & 0,1774 \\
France & $-0,03868$ & 16,785 & $-16,611$ & 0,1355 \\
Italie & $-0,0226$ & 27,0145 & $-26,544$ & 0,4479 \\
Luxembourg & 0,0853 & 8,4174 & $-7,7329$ & 0,7682 \\
\hline
\end{tabular}

Les trois premières colonnes de chaque ligne représentent respectivement, pour chacune des économies retenues, les valeurs moyennes des contributions des facteurs $A_{t-1}, B_{t}$ et $C_{t}$, la quatrième représente les valeurs moyennes des taux de croissance des taux de marge. La ligne consacrée aux 
États-Unis fournit donc les informations suivantes : au cours de la période 1963/2000 le taux de croissance annuel du taux de marge a été en moyenne de $0,21 \%$ puisque l'influence de $A_{t-1}$ tendait à le faire croître chaque année de $0,00579 \%$ en moyenne, celle de $B_{t}$ de $9,498 \%$ en moyenne alors que celle de $C_{t}$ tendait chaque année à le réduire de 9,294\% en moyenne. Les autres lignes fournissent évidemment des informations de même type mais relatives aux cinq autres économies. La faiblesse des contributions des $A_{t-1}$ dans chacune des cinq économies n'est pas surprenante. En effet, le taux de croissance TCTXM entre les instants $t-1$ et $t$ est uniquement déterminé par les stratégies dont les résultats vont déterminer le montant de $B_{t}$ et celui de $C_{t}$ compte tenu du montant du coût salarial par unité d'excédent brut d'exploitation $\left(A_{t-1}\right.$ ou $\left.A_{t-2}\right)$ hérité de la période $T-1$. Si $B_{t}=C_{t}=0, T C T X M_{t}$ est évidemment nul. Rappelons que le montant de $B_{t}$ et celui de $C_{t}$ dépendent des stratégies mises en oeuvre entre $t-1$ et $t$. Ces stratégies sont les stratégies de Mark-up, celles qui sont adoptées au cours des négociations salariales, les décisions d'embauche, leurs résultats dépendent de l'adhésion des salariés aux objectifs des directions et des degrés de validation des offres de biens et de services imposés par les marchés.

Les signes dont les contributions de $B_{t}$ et $C_{t}$ sont affectées sont bien conformes au fait que dans chacune de ces économies, le taux de croissance du taux de marge est une fonction croissante du pouvoir de détermination des prix et une fonction décroissante du taux de croissance du coût salarial unitaire. En prenant les valeurs absolues des moyennes portées dans les trois premières colonnes de chaque ligne et en inscrivant dans la quatrième la somme de ces moyennes, on obtient le tableau 2.

Tableau 2 : Contributions en valeur absolue des éléments $A, B$ et $C$

\begin{tabular}{lcccc}
\hline $1963 / 2000$ & $\left|\mathcal{C}^{A}\right|$ & $\left|\mathcal{C}^{B}\right|$ & $\left|\mathcal{C}^{C}\right|$ & Somme \\
\hline États-Unis & 0,00579 & 9,498 & 9,294 & 18,79779 \\
Allemagne & 0,026 & 8,801 & 8,4905 & 17,3175 \\
Royaume-Uni & 0,0884 & 13,527 & 13,438 & 27,0534 \\
France & 0,03868 & 16,785 & 16,611 & 33,43468 \\
Italie & 0,0226 & 27,0145 & 26,544 & 53,581 \\
Luxembourg & 0,0853 & 8,4174 & 7,7329 & 16,2356 \\
\hline
\end{tabular}

En divisant les termes de chacune des trois premières colonnes de chaque ligne du tableau 2 par la somme inscrite dans la quatrième colonne de cette ligne on obtient le tableau 3. 
Tableau 3 : Contributions en pourcentage entre 1963 et 2000

\begin{tabular}{lccc}
\hline $1963 / 2000$ & $\left|\mathcal{C}^{A}\right|$ en $\%$ & $\left|\mathcal{C}^{B}\right|$ en $\%$ & $\left|\mathcal{C}^{C}\right|$ en $\%$ \\
\hline États-Unis & 0,03 & 50,53 & 49,44 \\
Allemagne & 0,15 & 50,82 & 49,03 \\
Royaume-Uni & 0,13 & 50,22 & 49,65 \\
France & 0,12 & 50,2 & 49,68 \\
Italie & 0,04 & 50,42 & 49,54 \\
Luxembourg & 0,49 & 51,88 & 47,63 \\
\hline
\end{tabular}

Chacune des grandeurs contenues dans les colonnes de ce tableau exprime une influence. Dans la première il s'agit de celle du facteur $A_{t-1}$, dans la seconde de celle du facteur $B_{t}$, dans la troisième de celle du facteur $C_{t}$. Chacune de ces influences est exprimée en pourcentage de la somme des influences (les sommes de la quatrième colonne du tableau 2) qui ont contribué à déterminer le taux de croissance annuel moyen du taux de marge de chaque économie. Ainsi, la lecture de la première ligne permet de conclure que l'influence exercée par $A_{t-1}$ aux États-Unis a représenté beaucoup moins de $1 \%$ des influences qui ont contribué à déterminer le taux de croissance moyen annuel de son taux de marge, alors que l'influence de $B_{t}$ a représenté $50,53 \%$ de ces influences et celle de $C_{t} 49,44 \%$. Dans la mesure où le facteur $C_{t}$ n'est autre que le taux de croissance moyen annuel du coût salarial unitaire, ces informations permettent de classer les six économies en fonction de l'efficacité avec laquelle elles sont parvenues à cantonner, au sein des influences qui déterminaient le taux de croissance de leur taux de marge, celle que ce taux a exercé. Pour la période 1963/2000, ce classement, établi par ordre décroissant d'efficacité, est le suivant : Luxembourg, Allemagne, États-Unis, Italie, Royaume-Uni, France. Lorsque les moyennes inscrites dans les tableaux 1 et 2 sont calculées à propos de la période 1982/2000 au lieu de l'être à propos de la période 1963/2000, nous obtenons le tableau 4.

Tableau 4 : Contributions en pourcentage entre 1982 et 2000

\begin{tabular}{lccc}
\hline $1982 / 2000$ & $\left|\mathcal{C}^{A}\right|$ en $\%$ & $\left|\mathcal{C}^{B}\right|$ en $\%$ & $\left|\mathcal{C}^{C}\right|$ en $\%$ \\
\hline États-Unis & 0,02 & 53,55 & 46,43 \\
Allemagne & 0,33 & 59,03 & 40,64 \\
Royaume-Uni & 3,62 & 49,1 & 47,28 \\
France & 0,24 & 55,39 & 44,37 \\
Italie & 0,12 & 54,41 & 45,47 \\
Luxembourg & 0,09 & 63,06 & 36,85 \\
\hline
\end{tabular}


Le classement par ordre décroissant de maîtrise de l'influence exercée par le taux de croissance du coût salarial unitaire associé à ces résultats est donc le suivant : Luxembourg, Allemagne, France, Italie, États-Unis, Royaume-Uni.

\section{Conclusion}

Avec Blanchard $(1997,1998)$, qualifions d'anglo-saxonnes l'économie des États-Unis et celle du Royaume-Uni et de continentales celles d'Allemagne, de France, d'Italie et du Luxembourg. En tendance, au cours de la période 1961/2000, les évolutions des taux de chômage des premières peuvent être représentées à l'aide de paraboles ${ }^{12}$. L'une, celle qui concerne les États-Unis, passe par un maximum au tout début des années 1980 ; l'autre, celle qui concerne le Royaume-Uni, passe par un maximum au tout début des années 1990. Les ajustements polynomiaux associés aux graphes qui représentent les évolutions des taux de chômage des économies continentales sont au contraire des lieux de pentes croissantes sauf pour le Luxembourg à propos duquel l'ajustement est aussi une parabole (maximum en 1990). Comment expliquer que la tendance à la hausse des taux de chômage qui s'inverse dès le début des années 1980 aux États-Unis et dès le début des années 1990 au Royaume-Uni persiste dans les économies continentales? L'une des explications privilégiées pour répondre à cette question consiste à admettre que la rupture de tendance ne s'est pas produite dans les économies continentales car les rigidités de «leurs marchés du travail » les auraient empêchées d'ajuster, aussi bien que les économies anglo-saxonnes, le taux de croissance de leurs taux de salaire au ralentissement des gains de productivité amorcés à la fin des années 1960 ou au début des années 1970. L'existence de ces rigidités justifierait l'emploi de l'expression « eurosclérose ». Blanchard (2001, 2004), Boussemart et alii (2000), Freyssinet et alii (2000) sont peu convaincus par ce type d'analyse. Le premier n'hésite pas à parler de «la faiblesse des arguments en faveur de l'eurosclérose » (2001, p. 427), à qualifier les thèses selon lesquelles il suffirait d'éliminer les rigidités du « marché du travail », «de recettes simples, énoncées comme des conditions suffisantes » (2004, p. 3). Freyssinet et alii (2000, p. 26) notent que « les pays qui ont enregistré la plus forte progression du taux de chômage sur la période considérée $(1980 / 1998)$ sont également ceux qui ont fait preuve de la plus grande modération salariale mesurée par le rétablissement du taux de marge, c'est-à-dire de la part du profit dans la valeur ajoutée ». Ils concluent «ce lien profit-chômage représente un démenti pratique aux arguments avancés en faveur de la modération salariale comme condition préalable à un recul du chômage » (2000, p. 26). S'intéressant à l'évolution du rapport salaire réel/productivité

\footnotetext{
${ }^{12}$ Ces paraboles correspondent aux ajustements polynomiaux réalisés à partir des graphes qui retracent l'évolution des taux de chômage de ces deux économies. Ces graphes sont eux-mêmes obtenus en utilisant le tableau 3 (p. 130-131) de la source statistique identifiée au début de l'Annexe.
} 
globale des facteurs, Boussemart et alii (2000) notent que l'hypothèse de variation en sens opposés de ce rapport et du volume de l'emploi, si elle semble pertinente du début des années 1970 au début des années 1980, ne permet plus de comprendre les évolutions constatées après cette période. En effet, à partir du début des années 1980, le rapport salaire réel/productivité globale des facteurs ne cesse de croître au Royaume-Uni alors qu'il décroît en Allemagne, en France et en Italie. Or, le taux de chômage décroît au Royaume-Uni alors qu'il croît dans les autres économies. Ce constat incite les auteurs à conclure que le lien négatif entre le rapport salaire réel/productivité globale des facteurs et le niveau de l'emploi disparaît au début des années 1980 (2000, p. 17).

Les résultats que nous proposons dans le tableau 4 permettent de constater :

- qu'une très bonne maîtrise des coûts salariaux unitaires (Luxembourg) peut s'accompagner d'une dimnution du taux de chômage;

- qu'une bonne maîtrise des coûts salariaux unitaires (Allemagne, France, Italie) peut s'accompagner d'une forte augmentation du taux de chômage ;

- qu'une maîtrise des coûts salariaux unitaires moins bonnes que celles des quatre autres économies (États-unis, Royaume-uni) peut cependant s'accompagner d'une diminution du taux de chômage.

Ces résultats peuvent être considérés comme intéressants dans la mesure où ils confirment les conclusions auxquelles parviennent tous les auteurs que nous venons de citer. Nous pensons que leur intérêt est aussi lié à la valorisation de deux perspectives méthodologiques. La première est celle qui consiste à considérer la valeur de Shapley comme un outil qui, utilisé pour décomposer des agrégats, autorise une identification de la part de responsabilité de chacun des comportements dont on peut supposer qu'ils constituent «les forces» déterminant le montant de ces agrégats. La seconde est celle qui consiste à substituer à l'idée selon laquelle les rémunérations des apporteurs de capitaux dépendent de la productivité marginale des biens acquis grâce aux placements de leur épargne, celle selon laquelle elles sont la conséquence d'un prélèvement légal sur la valeur ajoutée. Cette idée, que Joan Robinson qualifiait de simple, permet nous semble-t-il d'introduire facilement dans l'analyse macroéconomique du fonctionnement des économies capitalistes, le rôle de l'Histoire à travers celui de l'évolution des rapports de force entre les détenteurs des rôles privés fondamentaux de ces économies. Les résultats auxquels ont conduit les controverses entre les Cambridge, ceux auxquels conduit l'emploi des fonctions distance afin de construire des fonctions de production ${ }^{13}$,

\footnotetext{
${ }^{13}$ L'emploi du concept de fonction distance (Briec (1997)) permet de supposer que la relation unissant les facteurs employés par une entreprise aux produits qu'elle offre n'est pas gouvernée par une forme fonctionnelle unique excluant la sous-utilisation du potentiel de production des facteurs. Or, l'observation des indicateurs de productivité globale des facteurs calculés en utilisant les fonctions distance conduit à conclure que, sauf exception, la rémunération du travail dans les industries manufacturières des principaux pays de l'OCDE n'est pas égale à sa productivité marginale
} 
semblent plaider en faveur de cette prise en compte.

\section{Bibliographie}

Auvray, C., et Trannoy, A. (1992), «Décomposition par source de l'inégalité des revenus à l'aide de la Valeur Shapley », Journées de Microéconomie Appliquée, Sfax, Tunisie.

Blanchard, O. (1997), «The Medium Run», Brookings Papers on Activity, vol. 2, pp. 89-157.

Blanchard, O. (1998), « Revisiting European Unemployment : Unemployment, Capital Accumulation and Factor Prices », NBER Working Paper, n W6566, May.

Blanchard, O. (2001), Macroéconomie, Pearson Education.

Blanchard, O. (2004), «Peut-on éliminer le chômage en Europe », Revue française d'économie, n4, vol. 18, pp. 1-31.

Boussemart, J-P (2002), « Productivité globale et rémunération des facteurs des industries manufacturières de l'OCDE », séminaire du GEREM, Perpignan.

Boussemart, J-P, Briec, W., et Poutineau, J-C (2000), «The Productivity Side of Eurosclerosis », North American Productivity Workshop, Schenectady New-York, June 15-17.

Briec, W. (1997), « A graph-type extension of Farrell technical efficiency measure », Journal of Productivity Analysis, vol. 8, pp. 95-110.

Cartelier, J. (1976), Surproduit et reproduction, Presses universitaires de Grenoble.

Chantreuil, F. et Trannoy, A., (1999), «Inequality Decomposition Values : The Trade-off Between Marginality and Consistency », DP 9924, THEMA.

Clower R. W. et Howitt P. (1995), «Les fondements de l'économie », dans d'Autume A. et Cartelier J. (1995), L'économie devient-elle une science dure?, pp. 18-35, Economica, Paris. .

Fleurbaey, M. (2006), Capitalisme ou démocratie? L'alternative du XXIe siècle, Grasset, Paris.

Freyssinet, J., Husson, M., Jolivet, A. et Tuchszirer, C. (2000), Les marchés du travail en Europe, Repères, La Découverte, Paris.

Guerrien, B. (1989), Concurrence, flexibilité et stabilité, Economica, Paris.

Keynes, J-M. (1968), Théorie générale de l'emploi, de l'intérêt et de la monnaie, Payot.

Lavoie, M. (1987), Macroéconomie : théorie et controverses post-keynésiennes, Dunod, Paris.

(Boussemart, 2002). 
Mussard, S. et Peypoch, N. (2006), « On Multi-Decomposition of the Aggregate Malmquist Productivity Index», Economics Letters, vol. 91, pp. 436-443.

Robinson, J. (1971), Essai sur l'économie de Marx, Dunod, Paris.

Sastre, M. et Trannoy, A. (2002), «Shapley inequality decomposition by factor components : Some methodological issues », dans Moyes, P., Seidl, C. et Shorrocks, A.F. (Ed.), Inequalities : Theory, Experiments and Applications, Journal of Economics, Supplément 9, pp. 51-90.

Shapley, L. (1953), « A value for n-person games », dans : Kuhn, H. W. et Tucker, A. W., (Ed.), Contributions to the Theory of Games, vol. 2, Princeton University Press.

Shorrocks, A.F. (1999), «Decomposition Procedures for Distributional Analysis : A Unified Framework Based on the Shapley Value », Mimeo, University of Essex.

Silber, J. (2004), «Inequalities : theory, experiments and applications », European Journal of Political Economy, vol. 20, pp. 813-820.

\section{Annexe : Mesures des déterminants des taux de croissance des taux de marge}

Les séries chronologiques de données annuelles utilisées sont celles que propose la Direction Générale des Affaires Économiques et Financières de la Commission Européenne. Les mesures A, $B$, et $C$ sont construites à partir de la part des salaires dans la valeur ajoutée $(C f$. Tableau 32 , p. 188-189), à partir de l'indice implicite des prix du PIB au prix de marché ( $C f$. Tableau 24, p. 172-173), à partir de la rémunération nominale par salarié pour l'ensemble de l'économie $(C f$. Tableau 29, p. 182-183), et enfin à partir du produit intérieur brut aux prix de 1995 par personne occupée ( $C f$. Tableau 11, p.146-147). Toutes ces séries sont fournies dans le numéro 72 de la Revue d'Économie Européenne.

La « vraie » mesure repose sur celle du taux de marge $(T X M)$; celle-ci est elle-même obtenue à partir de la série de la part des salaires dans la valeur ajoutée (Tableau 32, p.188-189 du n³2 de la Revue d'Économie Européenne) puisque, TXM étant égal à $\frac{V A-S}{V A}(C f$. équation (2)). La «vraie» mesure est donc donnée par $\frac{T X M_{t}-T X M_{t-1}}{T X M_{t-1}}$ et la mesure approchée par $A_{t-1}\left(B_{t}-C_{t}\right)$. 
Tableau A1 : USA

\begin{tabular}{|c|c|c|c|c|c|c|c|c|}
\hline $\begin{array}{c}\text { Composantes } \rightarrow \\
\text { Années } \downarrow\end{array}$ & $A_{t}^{14}$ & $B_{t}^{15}$ & $C_{t}^{16}$ & $\mathcal{C}^{A 17}$ & $\mathcal{C}^{B 18}$ & $\mathcal{C}^{C 19}$ & $A_{t-1}\left(B_{t}-C_{t}\right)^{20}$ & $T C T X M_{t}^{21,22}$ \\
\hline 1961 & 2,4247 & 0,0110 & 0,0050 & & & & & \\
\hline 1962 & 2,3445 & 0,0140 & 0,0050 & & & & & \\
\hline 1963 & 2,3113 & 0,0110 & 0,0050 & $-0,0002$ & 0,0262 & $-0,0119$ & 0,0141 & 0,0100 \\
\hline 1964 & 2,2680 & 0,0150 & 0,0110 & $-0,0001$ & 0,0349 & $-0,0256$ & 0,0092 & 0,0132 \\
\hline 1965 & 2,1546 & 0,0190 & 0,0060 & $-0,0003$ & 0,0435 & $-0,0137$ & 0,0295 & 0,0359 \\
\hline 1966 & 2,1250 & 0,0290 & 0,0300 & 0,0001 & 0,0641 & $-0,0663$ & $-0,0022$ & 0,0095 \\
\hline 1967 & 2,2258 & 0,0310 & 0,0430 & 0,0002 & 0,0663 & $-0,0920$ & $-0,0255$ & $-0,0313$ \\
\hline 1968 & 2,2787 & 0,0430 & 0,0500 & $-0,0004$ & 0,0935 & $-0,1088$ & $-0,0156$ & $-0,0161$ \\
\hline 1969 & 2,4364 & 0,0490 & 0,0680 & $-0,0005$ & 0,1104 & $-0,1532$ & $-0,0433$ & $-0,0459$ \\
\hline 1970 & 2,5587 & 0,0530 & 0,0660 & $-0,0010$ & 0,1250 & $-0,1556$ & $-0,0317$ & $-0,0344$ \\
\hline 1971 & 2,4247 & 0,0500 & 0,0340 & 0,0010 & 0,1249 & $-0,0849$ & 0,0409 & 0,0391 \\
\hline 1972 & 2,4014 & 0,0420 & 0,0430 & 0,0001 & 0,1047 & $-0,1071$ & $-0,0024$ & 0,0068 \\
\hline 1973 & 2,3670 & 0,0560 & 0,0540 & 0,0000 & 0,1351 & $-0,1303$ & 0,0048 & 0,0102 \\
\hline 1974 & 2,4843 & 0,0900 & 0,1030 & 0,0002 & 0,2146 & $-0,2456$ & $-0,0308$ & $-0,0337$ \\
\hline 1975 & 2,3113 & 0,0940 & 0,0730 & 0,0012 & 0,2280 & $-0,1771$ & 0,0522 & 0,0523 \\
\hline 1976 & 2,2680 & 0,0560 & 0,0560 & 0,0000 & 0,1343 & $-0,1343$ & 0,0000 & 0,0132 \\
\hline 1977 & 2,2573 & 0,0640 & 0,0650 & 0,0000 & 0,1465 & $-0,1488$ & $-0,0023$ & 0,0033 \\
\hline 1978 & 2,2258 & 0,0710 & 0,0720 & 0,0000 & 0,1606 & $-0,1629$ & $-0,0023$ & 0,0098 \\
\hline 1979 & 2,2258 & 0,0840 & 0,0890 & 0,0001 & 0,1883 & $-0,1995$ & $-0,0111$ & 0,0000 \\
\hline 1980 & 2,3333 & 0,0920 & 0,1060 & 0,0000 & 0,2048 & $-0,2359$ & $-0,0312$ & $-0,0323$ \\
\hline 1981 & 2,2573 & 0,0930 & 0,0800 & 0,0007 & 0,2120 & $-0,1824$ & 0,0303 & 0,0233 \\
\hline 1982 & 2,3784 & 0,0620 & 0,0810 & 0,0007 & 0,1423 & $-0,1859$ & $-0,0429$ & $-0,0358$ \\
\hline 1983 & 2,2154 & 0,0400 & 0,0200 & 0,0012 & 0,0927 & $-0,0464$ & 0,0476 & 0,0507 \\
\hline 1984 & 2,1447 & 0,0370 & 0,0280 & $-0,0007$ & 0,0850 & $-0,0643$ & 0,0199 & 0,0225 \\
\hline 1985 & 2,1447 & 0,0320 & 0,0320 & 0,0000 & 0,0698 & $-0,0698$ & 0,0000 & 0,0000 \\
\hline 1986 & 2,1546 & 0,0220 & 0,0240 & 0,0000 & 0,0472 & $-0,0515$ & $-0,0043$ & $-0,0031$ \\
\hline 1987 & 2,1949 & 0,0300 & 0,0380 & 0,0000 & 0,0645 & $-0,0817$ & $-0,0172$ & $-0,0126$ \\
\hline 1988 & 2,2154 & 0,0340 & 0,0360 & 0,0000 & 0,0739 & $-0,0783$ & $-0,0044$ & $-0,0064$ \\
\hline 1989 & 2,1056 & 0,0380 & 0,0220 & 0,0002 & 0,0838 & $-0,0485$ & 0,0354 & 0,0354 \\
\hline 1990 & 2,1546 & 0,0390 & 0,0460 & 0,0004 & 0,0843 & $-0,0994$ & $-0,0147$ & $-0,0155$ \\
\hline 1991 & 2,1949 & 0,0360 & 0,0400 & $-0,0001$ & 0,0767 & $-0,0852$ & $-0,0086$ & $-0,0126$ \\
\hline 1992 & 2,1646 & 0,0240 & 0,0210 & 0,0001 & 0,0522 & $-0,0457$ & 0,0066 & 0,0096 \\
\hline 1993 & 2,1447 & 0,0240 & 0,0220 & 0,0000 & 0,0523 & $-0,0480$ & 0,0043 & 0,0063 \\
\hline 1994 & 2,0864 & 0,0210 & 0,0090 & $-0,0001$ & 0,0452 & $-0,0194$ & 0,0257 & 0,0189 \\
\hline 1995 & 2,0488 & 0,0220 & 0,0160 & $-0,0002$ & 0,0465 & $-0,0338$ & 0,0125 & 0,0123 \\
\hline 1996 & 1,9674 & 0,0190 & 0,0080 & $-0,0002$ & 0,0393 & $-0,0165$ & 0,0225 & 0,0274 \\
\hline 1997 & 1,9155 & 0,0200 & 0,0110 & $-0,0004$ & 0,0402 & $-0,0221$ & 0,0177 & 0,0178 \\
\hline 1998 & 1,9674 & 0,0130 & 0,02300 &, 0003 & 0,0252 & $-0,0447$ & $-0,0192$ & $-0,0175$ \\
\hline 1999 & 1,9674 & 0,0150 & 0,0170 & $-0,0001$ & 0,0291 & $-0,0330$ & $-0,0039$ & 0,0000 \\
\hline 2000 & 1,9155 & 0,0210 & 0,0110 & 0,0000 & 0,0413 & $-0,0216$ & 0,0197 & 0,0178 \\
\hline
\end{tabular}

\footnotetext{
${ }^{14}$ Coût salarial moyen par unité de l'excédent brut d'exploitation dégagé par une unité de travail.

${ }^{15}$ Taux d'inflation.

${ }^{16}$ Taux de croissance du coût salarial unitaire.

${ }^{17}$ Contribution absolue de $A_{t-1}$ à $A_{t-1}\left(B_{t}-C_{t}\right)$.

${ }^{18}$ Contribution absolue de $B_{t}$ à $A_{t-1}\left(B_{t}-C_{t}\right)$.

${ }^{19}$ Contribution absolue de $C_{t}$ à $A_{t-1}\left(B_{t}-C_{t}\right)$.

${ }^{20}$ Taux de croissance du taux de marge estimé $=A_{t-1}\left(B_{t}-C_{t}\right)=\mathcal{C}^{A}+\mathcal{C}^{B}+\mathcal{C}^{C}$.

${ }^{21}$ Taux de croissance du taux de marge observé : $\frac{T X M_{t}-T X M_{t-1}}{T X M_{t}}$, où $T X M_{t}=1-\frac{s_{t}}{p_{t} P M R T_{t}}$.

${ }^{22}$ Il est aisé de vérifier la qualité de l'ajustement du graphique du taux de croissance du taux de marge calculé $A_{t-1}\left(B_{t}-C_{t}\right)$ au graphique du taux de croissance du taux de marge observé TCTX $M_{t}$.
} 
Tableau A2 : Allemagne

\begin{tabular}{|c|c|c|c|c|c|c|c|c|}
\hline $\begin{array}{c}\text { Composantes } \rightarrow \\
\text { Années } \downarrow\end{array}$ & $A_{t}$ & $B_{t}$ & $C_{t}$ & $\mathcal{C}^{A}$ & $\mathcal{C}^{B}$ & $\mathcal{C}^{C}$ & $A_{t-1}\left(B_{t}-C_{t}\right)$ & $T C T X M_{t}$ \\
\hline 1961 & 2,5842 & 0,0470 & 0,0700 & & & & & \\
\hline 1962 & 2,6364 & 0,0390 & 0,0480 & & & & & \\
\hline 1963 & 2,6496 & 0,0310 & 0,0350 & $-0,0001$ & 0,0809 & $-0,0914$ & $-0,0105$ & $-0,0036$ \\
\hline 1964 & 2,4965 & 0,0300 & 0,0160 & 0,0001 & 0,0793 & $-0,0423$ & 0,0371 & 0,0438 \\
\hline 1965 & 2,5211 & 0,0370 & 0,0470 & 0,0008 & 0,0952 & $-0,1209$ & $-0,0250$ & $-0,0070$ \\
\hline 1966 & 2,5971 & 0,0340 & 0,0450 & $-0,0001$ & 0,0853 & $-0,1129$ & $-0,0277$ & $-0,0211$ \\
\hline 1967 & 2,5088 & 0,0160 & 0,0030 & 0,0005 & 0,0409 & $-0,0077$ & 0,0338 & 0,0252 \\
\hline 1968 & 2,3333 & 0,0230 & 0,0130 & $-0,0004$ & 0,0587 & $-0,0332$ & 0,0251 & 0,0526 \\
\hline 1969 & 2,3898 & 0,0420 & 0,0370 & $-0,0004$ & 0,1017 & $-0,0896$ & 0,0117 & $-0,0167$ \\
\hline 1970 & 2,5842 & 0,0770 & 0,1230 & $-0,0013$ & 0,1818 & $-0,2905$ & $-0,1099$ & $-0,0542$ \\
\hline 1971 & 2,6630 & 0,0770 & 0,0880 & $-0,0011$ & 0,1915 & $-0,2189$ & $-0,0284$ & $-0,0215$ \\
\hline 1972 & 2,6765 & 0,0530 & 0,0580 & $-0,0002$ & 0,1391 & $-0,1522$ & $-0,0133$ & $-0,0037$ \\
\hline 1973 & 2,7879 & 0,0640 & 0,0830 & $-0,0001$ & 0,1709 & $-0,2216$ & $-0,0509$ & $-0,0294$ \\
\hline 1974 & 3,0323 & 0,0710 & 0,1000 & $-0,0016$ & 0,1940 & $-0,2732$ & $-0,0808$ & $-0,0606$ \\
\hline 1975 & 3,0000 & 0,0570 & 0,0550 & 0,0002 & 0,1659 & $-0,1601$ & 0,0061 & 0,0081 \\
\hline 1976 & 2,7879 & 0,0360 & 0,0180 & $-0,0003$ & 0,1086 & $-0,0543$ & 0,0540 & 0,0560 \\
\hline 1977 & 2,8023 & 0,0370 & 0,0390 & 0,0002 & 0,1071 & $-0,1129$ & $-0,0056$ & $-0,0038$ \\
\hline 1978 & 2,7037 & 0,0430 & 0,0330 & 0,0001 & 0,1202 & $-0,0922$ & 0,0280 & 0,0266 \\
\hline 1979 & 2,6630 & 0,0380 & 0,0330 & $-0,0002$ & 0,1046 & $-0,0908$ & 0,0135 & 0,0111 \\
\hline 1980 & 2,9216 & 0,0500 & 0,0740 & 0,0005 & 0,1342 & $-0,1986$ & $-0,0639$ & $-0,0659$ \\
\hline 1981 & 2,9683 & 0,0420 & 0,0460 & $-0,0005$ & 0,1173 & $-0,1284$ & $-0,0117$ & $-0,0118$ \\
\hline 1982 & 2,8911 & 0,0440 & 0,0390 & 0,0001 & 0,1296 & $-0,1149$ & 0,0148 & 0,0198 \\
\hline 1983 & 2,6101 & 0,0320 & 0,0040 & $-0,0011$ & 0,0937 & $-0,0117$ & 0,0809 & 0,0778 \\
\hline 1984 & 2,4843 & 0,0210 & 0,0080 & $-0,0018$ & 0,0578 & $-0,0220$ & 0,0339 & 0,0361 \\
\hline 1985 & 2,4130 & 0,0210 & 0,0160 & $-0,0003$ & 0,0535 & $-0,0408$ & 0,0124 & 0,0209 \\
\hline 1986 & 2,3333 & 0,0320 & 0,0270 & $-0,0002$ & 0,0784 & $-0,0661$ & 0,0121 & 0,0239 \\
\hline 1987 & 2,3784 & 0,0190 & 0,0250 & 0,0002 & 0,0451 & $-0,0593$ & $-0,0140$ & $-0,0133$ \\
\hline 1988 & 2,2573 & 0,0150 & 0,0010 & 0,0003 & 0,0353 & $-0,0024$ & 0,0333 & 0,0372 \\
\hline 1989 & 2,1746 & 0,0240 & 0,0080 & $-0,0010$ & 0,0556 & $-0,0185$ & 0,0361 & 0,0261 \\
\hline 1990 & 2,0960 & 0,0320 & 0,0200 & $-0,0005$ & 0,0709 & $-0,0443$ & 0,0261 & 0,0254 \\
\hline 1991 & 2,1746 & 0,0390 & 0,0340 & $-0,0002$ & 0,0833 & $-0,0726$ & 0,0105 & $-0,0248$ \\
\hline 1992 & 2,2895 & 0,0500 & 0,0670 & $-0,0007$ & 0,1068 & $-0,1431$ & $-0,0370$ & $-0,0349$ \\
\hline 1993 & 2,3223 & 0,0370 & 0,0380 & $-0,0001$ & 0,0826 & $-0,0848$ & $-0,0023$ & $-0,0099$ \\
\hline 1994 & 2,1949 & 0,0250 & 0,0050 & 0,0003 & 0,0576 & $-0,0115$ & 0,0464 & 0,0399 \\
\hline 1995 & 2,1646 & 0,0200 & 0,0210 & 0,0001 & 0,0452 & $-0,0474$ & $-0,0022$ & 0,0096 \\
\hline 1996 & 2,1153 & 0,0100 & 0,0020 & $-0,0001$ & 0,0218 & $-0,0044$ & 0,0173 & 0,0158 \\
\hline 1997 & 2,0211 & 0,0080 & $-0,0080$ & $-0,0004$ & 0,0171 & 0,0171 & 0,0338 & 0,0312 \\
\hline 1998 & 1,9586 & 0,0110 & 0,0000 & $-0,0005$ & 0,0228 & 0,0000 & 0,0222 & 0,0211 \\
\hline 1999 & 1,9851 & 0,0090 & 0,0060 & $-0,0001$ & 0,0179 & $-0,0119$ & 0,0059 & $-0,0089$ \\
\hline 2000 & 1,9762 & $-0,0040$ & $-0,0020$ & 0,0000 & $-0,0079$ & 0,0039 & $-0,0040$ & 0,0030 \\
\hline
\end{tabular}


Tableau A3 : Royaume-Uni

\begin{tabular}{|c|c|c|c|c|c|c|c|c|}
\hline $\begin{array}{c}\text { Composantes } \rightarrow \\
\text { Années } \downarrow\end{array}$ & $A_{t}$ & $B_{t}$ & $C_{t}$ & $\mathcal{C}^{A}$ & $\mathcal{C}^{B}$ & $\mathcal{C}^{C}$ & $A_{t-1}\left(B_{t}-C_{t}\right)$ & $T C T X M_{t}$ \\
\hline 1961 & 2,7175 & 0,0320 & 0,0540 & & & & & \\
\hline 1962 & 2,8023 & 0,0350 & 0,0410 & & & & & \\
\hline 1963 & 2,6101 & 0,0210 & 0,0040 & 0,0007 & 0,0580 & $-0,0110$ & 0,0476 & 0,0532 \\
\hline 1964 & 2,5587 & 0,0360 & 0,0270 & $-0,0009$ & 0,0974 & $-0,0731$ & 0,0235 & 0,0144 \\
\hline 1965 & 2,6101 & 0,0500 & 0,0520 & 0,0001 & 0,1292 & $-0,1344$ & $-0,0051$ & $-0,0142$ \\
\hline 1966 & 2,7175 & 0,0440 & 0,0510 & $-0,0002$ & 0,1137 & $-0,1318$ & $-0,0183$ & $-0,0289$ \\
\hline 1967 & 2,6765 & 0,0300 & 0,0240 & 0,0003 & 0,0799 & $-0,0639$ & 0,0163 & 0,0112 \\
\hline 1968 & 2,6232 & 0,0410 & 0,0300 & $-0,0002$ & 0,1106 & $-0,0809$ & 0,0294 & 0,0147 \\
\hline 1969 & 2,7313 & 0,0550 & 0,0520 & $-0,0001$ & 0,1457 & $-0,1378$ & 0,0079 & $-0,0290$ \\
\hline 1970 & 2,9683 & 0,0740 & 0,1010 & $-0,0015$ & 0,1981 & $-0,2704$ & $-0,0737$ & $-0,0597$ \\
\hline 1971 & 2,7037 & 0,0930 & 0,0840 & 0,0011 & 0,2650 & $-0,2394$ & 0,0267 & 0,0714 \\
\hline 1972 & 2,6765 & 0,0810 & 0,0930 & 0,0016 & 0,2297 & $-0,2637$ & $-0,0324$ & 0,0074 \\
\hline 1973 & 2,5971 & 0,0720 & 0,0780 & 0,0001 & 0,1937 & $-0,2098$ & $-0,0161$ & 0,0221 \\
\hline 1974 & 2,9841 & 0,1500 & 0,2100 & 0,0024 & 0,3955 & $-0,5537$ & $-0,1558$ & $-0,0971$ \\
\hline 1975 & 3,4643 & 0,2710 & 0,3180 & $-0,0091$ & 0,7563 & $-0,8874$ & $-0,1403$ & $-0,1076$ \\
\hline 1976 & 3,0000 & 0,1520 & 0,1110 & 0,0098 & 0,4901 & $-0,3579$ & 0,1420 & 0,1161 \\
\hline 1977 & 2,5971 & 0,1380 & 0,0840 & $-0,0125$ & 0,4460 & $-0,2715$ & 0,1620 & 0,1120 \\
\hline 1978 & 2,5336 & 0,1160 & 0,1100 & $-0,0012$ & 0,3246 & $-0,3078$ & 0,0156 & 0,0180 \\
\hline 1979 & 2,6364 & 0,1450 & 0,1400 & $-0,0002$ & 0,3720 & $-0,3591$ & 0,0127 & $-0,0283$ \\
\hline 1980 & 2,9370 & 0,1940 & 0,2180 & $-0,0012$ & 0,5015 & $-0,5635$ & $-0,0633$ & $-0,0764$ \\
\hline 1981 & 3,0161 & 0,1130 & 0,1140 & $-0,0002$ & 0,3149 & $-0,3177$ & $-0,0029$ & $-0,0197$ \\
\hline 1982 & 2,7594 & 0,0740 & 0,0480 & 0,0010 & 0,2203 & $-0,1429$ & 0,0784 & 0,0683 \\
\hline 1983 & 2,5461 & 0,0530 & 0,0360 & $-0,0022$ & 0,1530 & $-0,1040$ & 0,0469 & 0,0602 \\
\hline 1984 & 2,6101 & 0,0460 & 0,0550 & 0,0010 & 0,1220 & $-0,1459$ & $-0,0229$ & $-0,0177$ \\
\hline 1985 & 2,5336 & 0,0560 & 0,0500 & 0,0002 & 0,1444 & $-0,1289$ & 0,0157 & 0,0217 \\
\hline 1986 & 2,6630 & 0,0310 & 0,0370 & 0,0002 & 0,0797 & $-0,0952$ & $-0,0152$ & $-0,0353$ \\
\hline 1987 & 2,6232 & 0,0520 & 0,0490 & 0,0002 & 0,1351 & $-0,1273$ & 0,0080 & 0,0110 \\
\hline 1988 & 2,6900 & 0,0600 & 0,0670 & 0,0001 & 0,1586 & $-0,1771$ & $-0,0184$ & $-0,0181$ \\
\hline 1989 & 2,8911 & 0,0750 & 0,0990 & $-0,0008$ & 0,1992 & $-0,2630$ & $-0,0646$ & $-0,0517$ \\
\hline 1990 & 3,0816 & 0,0770 & 0,0950 & $-0,0018$ & 0,2149 & $-0,2651$ & $-0,0520$ & $-0,0467$ \\
\hline 1991 & 3,2735 & 0,0670 & 0,0740 & $-0,0007$ & 0,2001 & $-0,2210$ & $-0,0216$ & $-0,0449$ \\
\hline 1992 & 3,0816 & 0,0400 & 0,0280 & 0,0012 & 0,1271 & $-0,0890$ & 0,0393 & 0,0470 \\
\hline 1993 & 2,8168 & 0,0270 & 0,0060 & $-0,0020$ & 0,0858 & $-0,0191$ & 0,0647 & 0,0694 \\
\hline 1994 & 2,6496 & 0,0150 & $-0,0020$ & $-0,0023$ & 0,0442 & 0,0059 & 0,0479 & 0,0458 \\
\hline 1995 & 2,5842 & 0,0250 & 0,0140 & $-0,0009$ & 0,0683 & $-0,0383$ & 0,0291 & 0,0182 \\
\hline 1996 & 2,4843 & 0,0330 & 0,0230 & $-0,0003$ & 0,0864 & $-0,0602$ & 0,0258 & 0,0287 \\
\hline 1997 & 2,5088 & 0,0290 & 0,0290 & 0,0000 & 0,0735 & $-0,0735$ & 0,0000 & $-0,0070$ \\
\hline 1998 & 2,5714 & 0,0300 & 0,0370 & $-0,0001$ & 0,0749 & $-0,0924$ & $-0,0176$ & $-0,0175$ \\
\hline 1999 & 2,7594 & 0,0230 & 0,0400 & $-0,0005$ & 0,0584 & $-0,1016$ & $-0,0437$ & $-0,0500$ \\
\hline 2000 & 2,8168 & 0,0180 & 0,0210 & $-0,0003$ & 0,0480 & $-0,0560$ & $-0,0083$ & $-0,0150$ \\
\hline
\end{tabular}


Tableau A4 : France

\begin{tabular}{|c|c|c|c|c|c|c|c|c|}
\hline $\begin{array}{c}\text { Composantes } \rightarrow \\
\text { Années } \downarrow\end{array}$ & $A_{t}$ & $B_{t}$ & $C_{t}$ & $\mathcal{C}^{A}$ & $\mathcal{C}^{B}$ & $\mathcal{C}^{C}$ & $A_{t-1}\left(B_{t}-C_{t}\right)$ & $T C T X M_{t}$ \\
\hline 1961 & 2,9841 & 0,0270 & 0,0520 & & & & & \\
\hline 1962 & 3,0000 & 0,0520 & 0,0510 & & & & & \\
\hline 1963 & 3,0816 & 0,0660 & 0,0710 & 0,0000 & 0,1975 & $-0,2124$ & $-0,0150$ & $-0,0200$ \\
\hline 1964 & 3,0650 & 0,0410 & 0,0380 & 0,0001 & 0,1247 & $-0,1156$ & 0,0092 & 0,0041 \\
\hline 1965 & 2,9370 & 0,0300 & 0,0210 & $-0,0001$ & 0,0922 & $-0,0645$ & 0,0276 & 0,0325 \\
\hline 1966 & 2,7879 & 0,0300 & 0,0160 & $-0,0009$ & 0,0900 & $-0,0480$ & 0,0411 & 0,0394 \\
\hline 1967 & 2,6765 & 0,0320 & 0,0260 & $-0,0004$ & 0,0916 & $-0,0744$ & 0,0167 & 0,0303 \\
\hline 1968 & 2,8462 & 0,0400 & 0,0730 & 0,0018 & 0,1093 & $-0,1994$ & $-0,0883$ & $-0,0441$ \\
\hline 1969 & 2,7453 & 0,0690 & 0,0550 & 0,0012 & 0,1905 & $-0,1519$ & 0,0398 & 0,0269 \\
\hline 1970 & 2,7175 & 0,0550 & 0,0620 & 0,0004 & 0,1538 & $-0,1733$ & $-0,0192$ & 0,0075 \\
\hline 1971 & 2,7313 & 0,0630 & 0,0700 & 0,0001 & 0,1721 & $-0,1912$ & $-0,0190$ & $-0,0037$ \\
\hline 1972 & 2,6496 & 0,0700 & 0,0630 & 0,0000 & 0,1907 & $-0,1716$ & 0,0191 & 0,0224 \\
\hline 1973 & 2,5842 & 0,0850 & 0,0840 & 0,0000 & 0,2287 & $-0,2260$ & 0,0026 & 0,0182 \\
\hline 1974 & 2,8462 & 0,1180 & 0,1560 & 0,0012 & 0,3088 & $-0,4082$ & $-0,0982$ & $-0,0681$ \\
\hline 1975 & 3,3668 & 0,1300 & 0,1810 & $-0,0067$ & 0,3530 & $-0,4914$ & $-0,1452$ & $-0,1192$ \\
\hline 1976 & 3,4248 & 0,1110 & 0,1140 & $-0,0008$ & 0,3448 & $-0,3541$ & $-0,0101$ & $-0,0131$ \\
\hline 1977 & 3,3290 & 0,0930 & 0,0980 & $-0,0001$ & 0,3158 & $-0,3328$ & $-0,0171$ & 0,0221 \\
\hline 1978 & 3,2735 & 0,1010 & 0,0960 & $-0,0002$ & 0,3411 & $-0,3242$ & 0,0166 & 0,0130 \\
\hline 1979 & 3,3478 & 0,1000 & 0,0990 & 0,0000 & 0,3301 & $-0,3268$ & 0,0033 & $-0,0171$ \\
\hline 1980 & 3,4843 & 0,1110 & 0,1290 & $-0,0007$ & 0,3675 & $-0,4271$ & $-0,0603$ & $-0,0304$ \\
\hline 1981 & 3,6083 & 0,1100 & 0,1220 & $-0,0008$ & 0,3758 & $-0,4168$ & $-0,0418$ & $-0,0269$ \\
\hline 1982 & 3,6296 & 0,1150 & 0,1170 & $-0,0001$ & 0,4078 & $-0,4149$ & $-0,0072$ & $-0,0046$ \\
\hline 1983 & 3,4843 & 0,0900 & 0,0830 & 0,0001 & 0,3257 & $-0,3004$ & 0,0254 & 0,0324 \\
\hline 1984 & 3,2735 & 0,0700 & 0,0540 & $-0,0012$ & 0,2490 & $-0,1921$ & 0,0557 & 0,0493 \\
\hline 1985 & 3,1667 & 0,0540 & 0,0450 & $-0,0009$ & 0,1825 & $-0,1521$ & 0,0295 & 0,0256 \\
\hline 1986 & 2,7879 & 0,0510 & 0,0220 & $-0,0015$ & 0,1642 & $-0,0708$ & 0,0918 & 0,1000 \\
\hline 1987 & 2,6630 & 0,0290 & 0,0140 & $-0,0028$ & 0,0863 & $-0,0417$ & 0,0418 & 0,0341 \\
\hline 1988 & 2,4843 & 0,0300 & 0,0070 & $-0,0014$ & 0,0818 & $-0,0191$ & 0,0612 & 0,0513 \\
\hline 1989 & 2,3445 & 0,0310 & 0,0160 & $-0,0013$ & 0,0798 & $-0,0412$ & 0,0373 & 0,0418 \\
\hline 1990 & 2,3784 & 0,0290 & 0,0340 & 0,0003 & 0,0700 & $-0,0821$ & $-0,0117$ & $-0,0100$ \\
\hline 1991 & 2,3670 & 0,0300 & 0,0310 & 0,0000 & 0,0708 & $-0,0732$ & $-0,0024$ & 0,0034 \\
\hline 1992 & 2,3445 & 0,0200 & 0,0210 & 0,0000 & 0,0475 & $-0,0498$ & $-0,0024$ & 0,0067 \\
\hline 1993 & 2,3445 & 0,0230 & 0,0220 & 0,0000 & 0,0542 & $-0,0518$ & 0,0023 & 0,0000 \\
\hline 1994 & 2,2573 & 0,0170 & $-0,0030$ & 0,0000 & 0,0399 & 0,0070 & 0,0469 & 0,0268 \\
\hline 1995 & 2,2573 & 0,0170 & 0,0140 & $-0,0001$ & 0,0391 & $-0,0322$ & 0,0068 & 0,0000 \\
\hline 1996 & 2,3003 & 0,0140 & 0,0140 & 0,0000 & 0,0316 & $-0,0316$ & 0,0000 & $-0,0130$ \\
\hline 1997 & 2,2468 & 0,0130 & 0,0070 & 0,0001 & 0,0296 & $-0,0160$ & 0,0138 & 0,0165 \\
\hline 1998 & 2,2258 & 0,0090 & 0,0050 & $-0,0001$ & 0,0205 & $-0,0114$ & 0,0090 & 0,0065 \\
\hline 1999 & 2,2573 & 0,0040 & 0,0090 & 0,0001 & 0,0089 & $-0,0201$ & $-0,0111$ & $-0,0097$ \\
\hline 2000 & 2,2258 & 0,0050 & 0,0040 & 0,0000 & 0,0112 & $-0,0090$ & 0,0023 & 0,0098 \\
\hline
\end{tabular}


Tableau A5 : Italie

\begin{tabular}{|c|c|c|c|c|c|c|c|c|}
\hline $\begin{array}{c}\text { Composantes } \rightarrow \\
\text { Années } \downarrow\end{array}$ & $A_{t}$ & $B_{t}$ & $C_{t}$ & $\mathcal{C}^{A}$ & $\mathcal{C}^{B}$ & $\mathcal{C}^{C}$ & $A_{t-1}\left(B_{t}-C_{t}\right)$ & $T C T X M_{t}$ \\
\hline 1961 & 2,9841 & 0,0280 & 0,0020 & & & & & \\
\hline 1962 & 2,9370 & 0,0580 & 0,0610 & & & & & \\
\hline 1963 & 3,2735 & 0,0850 & 0,1240 & 0,0009 & 0,2516 & $-0,3671$ & $-0,1145$ & $-0,0787$ \\
\hline 1964 & 3,4643 & 0,0650 & 0,0830 & $-0,0030$ & 0,2018 & $-0,2577$ & $-0,0589$ & $-0,0427$ \\
\hline 1965 & 3,2735 & 0,0420 & 0,0310 & 0,0010 & 0,1415 & $-0,1044$ & 0,0381 & 0,0446 \\
\hline 1966 & 3,0000 & 0,0220 & 0,0030 & $-0,0018$ & 0,0741 & $-0,0101$ & 0,0622 & 0,0684 \\
\hline 1967 & 3,0000 & 0,0280 & 0,0240 & $-0,0005$ & 0,0878 & $-0,0753$ & 0,0120 & 0,0000 \\
\hline 1968 & 2,8168 & 0,0170 & 0,0090 & 0,0000 & 0,0510 & $-0,0270$ & 0,0240 & 0,0480 \\
\hline 1969 & 2,5714 & 0,0410 & 0,0200 & $-0,0019$ & 0,1192 & $-0,0582$ & 0,0592 & 0,0687 \\
\hline 1970 & 2,8314 & 0,0690 & 0,1020 & 0,0040 & 0,1859 & $-0,2748$ & $-0,0849$ & $-0,0679$ \\
\hline 1971 & 3,2735 & 0,0690 & 0,1170 & $-0,0062$ & 0,1864 & $-0,3161$ & $-0,1359$ & $-0,1034$ \\
\hline 1972 & 3,2553 & 0,0600 & 0,0690 & $-0,0020$ & 0,1831 & $-0,2106$ & $-0,0295$ & 0,0043 \\
\hline 1973 & 3,1667 & 0,1300 & 0,1320 & 0,0000 & 0,4244 & $-0,4309$ & $-0,0065$ & 0,0213 \\
\hline 1974 & 3,0486 & 0,2010 & 0,1970 & $-0,0002$ & 0,6454 & $-0,6326$ & 0,0127 & 0,0292 \\
\hline 1975 & 3,5455 & 0,1650 & 0,2320 & 0,0040 & 0,5128 & $-0,7210$ & $-0,2043$ & $-0,1093$ \\
\hline 1976 & 3,3668 & 0,1790 & 0,1620 & 0,0042 & 0,5902 & $-0,5341$ & 0,0603 & 0,0409 \\
\hline 1977 & 3,4843 & 0,1870 & 0,1930 & 0,0005 & 0,6463 & $-0,6670$ & $-0,0202$ & $-0,0262$ \\
\hline 1978 & 3,3668 & 0,1350 & 0,1330 & 0,0001 & 0,4625 & $-0,4556$ & 0,0070 & 0,0269 \\
\hline 1979 & 3,1667 & 0,1590 & 0,1590 & 0,0000 & 0,5447 & $-0,5447$ & 0,0000 & 0,0480 \\
\hline 1980 & 3,0650 & 0,2140 & 0,2020 & $-0,0012$ & 0,6991 & $-0,6599$ & 0,0380 & 0,0250 \\
\hline 1981 & 3,2918 & 0,1900 & 0,2170 & 0,0014 & 0,5920 & $-0,6761$ & $-0,0828$ & $-0,0528$ \\
\hline 1982 & 3,2194 & 0,1720 & 0,1600 & 0,0014 & 0,5467 & $-0,5086$ & 0,0395 & 0,0172 \\
\hline 1983 & 3,3290 & 0,1510 & 0,1520 & 0,0000 & 0,4916 & $-0,4949$ & $-0,0032$ & $-0,0253$ \\
\hline 1984 & 3,0323 & 0,1150 & 0,0930 & 0,0012 & 0,3765 & $-0,3045$ & 0,0732 & 0,0736 \\
\hline 1985 & 2,9063 & 0,0890 & 0,0790 & $-0,0015$ & 0,2831 & $-0,2513$ & 0,0303 & 0,0323 \\
\hline 1986 & 2,6900 & 0,0790 & 0,0580 & $-0,0013$ & 0,2346 & $-0,1722$ & 0,0610 & 0,0586 \\
\hline 1987 & 2,6765 & 0,0620 & 0,0540 & $-0,0009$ & 0,1735 & $-0,1511$ & 0,0215 & 0,0037 \\
\hline 1988 & 2,6364 & 0,0680 & 0,0530 & $-0,0001$ & 0,1825 & $-0,1422$ & 0,0401 & 0,0110 \\
\hline 1989 & 2,5842 & 0,0650 & 0,0600 & $-0,0001$ & 0,1727 & $-0,1594$ & 0,0132 & 0,0145 \\
\hline 1990 & 2,7736 & 0,0820 & 0,0940 & 0,0003 & 0,2140 & $-0,2454$ & $-0,0310$ & $-0,0502$ \\
\hline 1991 & 2,8760 & 0,0760 & 0,0820 & $-0,0006$ & 0,2036 & $-0,2197$ & $-0,0166$ & $-0,0264$ \\
\hline 1992 & 2,8760 & 0,0450 & 0,0440 & 0,0001 & 0,1271 & $-0,1243$ & 0,0029 & 0,0000 \\
\hline 1993 & 2,7736 & 0,0390 & 0,0240 & 0,0000 & 0,1122 & $-0,0690$ & 0,0431 & 0,0271 \\
\hline 1994 & 2,4602 & 0,0350 & $-0,0020$ & $-0,0019$ & 0,0989 & 0,0056 & 0,1026 & 0,0906 \\
\hline 1995 & 2,2154 & 0,0500 & 0,0130 & $-0,0058$ & 0,1308 & $-0,0340$ & 0,0910 & 0,0761 \\
\hline 1996 & 2,1847 & 0,0530 & 0,0530 & 0,0000 & 0,1239 & $-0,1239$ & 0,0000 & 0,0096 \\
\hline 1997 & 2,2362 & 0,0240 & 0,0240 & 0,0000 & 0,0528 & $-0,0528$ & 0,0000 & $-0,0159$ \\
\hline 1998 & 2,1250 & 0,0270 & $-0,0230$ & 0,0013 & 0,0597 & 0,0508 & 0,1118 & 0,0356 \\
\hline 1999 & 2,1153 & 0,0160 & 0,0160 & 0,0000 & 0,0349 & $-0,0349$ & 0,0000 & 0,0031 \\
\hline 2000 & 2,0675 & 0,0220 & 0,0150 & 0,0000 & 0,0466 & $-0,0318$ & 0,0148 & 0,0156 \\
\hline
\end{tabular}


Tableau A6 : Luxembourg

\begin{tabular}{|c|c|c|c|c|c|c|c|c|}
\hline $\begin{array}{c}\text { Composantes } \rightarrow \\
\text { Années } \downarrow\end{array}$ & $A_{t}$ & $B_{t}$ & $C_{t}$ & $\mathcal{C}^{A}$ & $\mathcal{C}^{B}$ & $\mathcal{C}^{C}$ & $A_{t-1}\left(B_{t}-C_{t}\right)$ & $T C T X M_{t}$ \\
\hline 1961 & 1,4155 & $-0,0370$ & 0,0020 & & & & & \\
\hline 1962 & 1,3866 & 0,0390 & 0,0370 & & & & & \\
\hline 1963 & 1,4096 & 0,0310 & 0,0420 & 0,0002 & 0,0434 & $-0,0588$ & $-0,0153$ & $-0,0095$ \\
\hline 1964 & 1,4155 & 0,0580 & 0,0730 & $-0,0002$ & 0,0811 & $-0,1021$ & $-0,0211$ & $-0,0024$ \\
\hline 1965 & 1,4450 & 0,0280 & 0,0320 & 0 & 0,0396 & $-0,0452$ & $-0,0057$ & $-0,0121$ \\
\hline 1966 & 1,4450 & 0,0390 & 0,0440 & $-0,0001$ & 0,0558 & $-0,0629$ & $-0,0072$ & 0 \\
\hline 1967 & 1,4876 & 0,0040 & 0,0150 & 0 & 0,0058 & $-0,0217$ & $-0,0159$ & $-0,0171$ \\
\hline 1968 & 1,3585 & 0,0500 & 0,0130 & 0,0008 & 0,0733 & $-0,0191$ & 0,055 & 0,05473 \\
\hline 1969 & 1,1459 & 0,0530 & $-0,0290$ & $-0,0053$ & 0,0754 & 0,0413 & 0,1114 & 0,09906 \\
\hline 1970 & 1,1692 & 0,1510 & 0,1540 & 0,0003 & 0,1891 & $-0,1928$ & $-0,0034$ & $-0,0107$ \\
\hline 1971 & 1,4691 & $-0,0080$ & 0,0830 & $-0,0011$ & $-0,0093$ & $-0,0961$ & $-0,1064$ & $-0,1215$ \\
\hline 1972 & 1,4938 & 0,0580 & 0,0590 & $-0,0001$ & 0,0765 & $-0,0778$ & $-0,0015$ & $-0,0099$ \\
\hline 1973 & 1,2624 & 0,1220 & 0,0510 & 0,0009 & 0,1807 & $-0,0756$ & 0,1061 & 0,1022 \\
\hline 1974 & 1,3310 & 0,1700 & 0,2150 & 0,0052 & 0,2343 & $-0,2963$ & $-0,0568$ & $-0,0294$ \\
\hline 1975 & 2,4722 & $-0,0090$ & 0,2010 & $-0,0072$ & $-0,0117$ & $-0,2606$ & $-0,2795$ & $-0,3287$ \\
\hline 1976 & 2,1250 & 0,1220 & 0,0840 & 0,0217 & 0,232 & $-0,1597$ & 0,0939 & 0,1111 \\
\hline 1977 & 2,6630 & 0,0120 & 0,0830 & 0,0123 & 0,0276 & $-0,1908$ & $-0,1509$ & $-0,1469$ \\
\hline 1978 & 2,3784 & 0,0510 & 0,0120 & 0,0105 & 0,1221 & $-0,0287$ & 0,1039 & 0,0842 \\
\hline 1979 & 2,2468 & 0,0640 & 0,0490 & $-0,0021$ & 0,1613 & $-0,1235$ & 0,0357 & 0,0405 \\
\hline 1980 & 2,4247 & 0,0790 & 0,0910 & 0,0008 & 0,1827 & $-0,2104$ & $-0,027$ & $-0,0519$ \\
\hline 1981 & 2,5211 & 0,0720 & 0,0920 & $-0,0018$ & 0,1682 & $-0,2149$ & $-0,0485$ & $-0,0274$ \\
\hline 1982 & 2,1949 & 0,1080 & 0,0550 & 0,0026 & 0,2671 & $-0,136$ & 0,1336 & 0,1021 \\
\hline 1983 & 2,1056 & 0,0680 & 0,0360 & $-0,0052$ & 0,1603 & $-0,0849$ & 0,0702 & 0,0287 \\
\hline 1984 & 1,9762 & 0,0440 & 0,0150 & $-0,0013$ & 0,0946 & $-0,0323$ & 0,0611 & 0,0435 \\
\hline 1985 & 1,9674 & 0,0300 & 0,0230 & $-0,0005$ & 0,0612 & $-0,0469$ & 0,0138 & 0,0030 \\
\hline 1986 & 1,8169 & 0,0280 & 0,0060 & $-0,0001$ & 0,0552 & $-0,0118$ & 0,0433 & 0,0534 \\
\hline 1987 & 2,0030 & 0,0090 & 0,0440 & 0,0026 & 0,017 & $-0,0833$ & $-0,0636$ & $-0,0620$ \\
\hline 1988 & 1,7778 & 0,0070 & $-0,0380$ & 0,0042 & 0,0134 & 0,0726 & 0,0901 & 0,0811 \\
\hline 1989 & 1,7174 & 0,0350 & 0,0160 & $-0,0021$ & 0,0662 & $-0,0302$ & 0,0338 & 0,0222 \\
\hline 1990 & 1,9070 & 0,0340 & 0,0710 & 0,0011 & 0,0594 & $-0,1241$ & $-0,0635$ & $-0,0652$ \\
\hline 1991 & 2,0864 & 0,0150 & 0,0450 & $-0,0028$ & 0,0272 & $-0,0815$ & $-0,0572$ & $-0,0581$ \\
\hline 1992 & 2,0488 & 0,0430 & 0,0340 & 0,0008 & 0,0859 & $-0,0679$ & 0,0188 & 0,0123 \\
\hline 1993 & 1,9762 & 0,0070 & $-0,0140$ & $-0,0004$ & 0,0145 & 0,0289 & 0,043 & 0,0243 \\
\hline 1994 & 1,8169 & 0,0530 & 0,0240 & $-0,0011$ & 0,1067 & $-0,0483$ & 0,0573 & 0,0565 \\
\hline 1995 & 1,8736 & 0,0070 & 0,0100 & 0,0002 & 0,0133 & $-0,019$ & $-0,0055$ & $-0,0197$ \\
\hline 1996 & 1,8818 & 0,0170 & 0,0210 & $-0,0001$ & 0,0314 & $-0,0387$ & $-0,0075$ & $-0,0029$ \\
\hline 1997 & 1,7027 & 0,0330 & $-0,0090$ & 0,0002 & 0,062 & 0,0169 & 0,079 & 0,0663 \\
\hline 1998 & 1,6455 & 0,0150 & 0,0030 & $-0,0011$ & 0,0269 & $-0,0054$ & 0,0204 & 0,0216 \\
\hline 1999 & 1,7624 & 0,0230 & 0,0070 & $-0,0005$ & 0,0385 & $-0,0117$ & 0,0263 & $-0,0423$ \\
\hline 2000 & 1,7100 & 0,0410 & 0,0230 & 0,0011 & 0,0699 & $-0,0392$ & 0,0317 & 0,0193 \\
\hline
\end{tabular}

